\title{
An uncleavable form of pro-scatter factor suppresses tumor growth and dissemination in mice
}

\author{
Massimiliano Mazzone, ${ }^{1}$ Cristina Basilico, ${ }^{1}$ Silvia Cavassa, ${ }^{1}$ Selma Pennacchietti, ${ }^{1}$ \\ Mauro Risio, ${ }^{2}$ Luigi Naldini, ${ }^{3}$ Paolo M. Comoglio, ${ }^{1}$ and Paolo Michieli ${ }^{1}$ \\ 1Division of Molecular Oncology, ${ }^{2}$ Unit of Pathological Anatomy, and ' 2 Laboratory of Gene Transfer and Therapy, \\ Institute for Cancer Research and Treatment, University of Torino Medical School, Candiolo, Italy.
}

\begin{abstract}
Scatter factor (SF), also known as hepatocyte growth factor, is ubiquitously present in the extracellular matrix of tissues in the form of an inactive precursor (pro-SF). In order to acquire biological activity, pro-SF must be cleaved by specific proteases present on the cell surface. The mature form of SF controls invasive cues in both physiological and pathological processes through activation of its receptor, the Met tyrosine kinase. By substituting a single amino acid in the proteolytic site, we engineered an unprocessable form of pro-SF (uncleavable SF). Using lentivirus vector technology, we achieved local or systemic delivery of uncleavable SF in mice. We provide evidence that (a) uncleavable SF inhibits both protease-mediated pro-SF conversion and active SF-induced Met activation; (b) local expression of uncleavable SF in tumors suppresses tumor growth, impairs tumor angiogenesis, and prevents metastatic dissemination; and (c) systemic expression of uncleavable SF dramatically inhibits the growth of transplanted tumors and abolishes the formation of spontaneous metastases without perturbing vital physiological functions. These data show that proteolytic activation of pro-SF is a limiting step in tumor progression, thus suggesting a new strategy for the treatment or prevention of the malignant conversion of neoplastic lesions.
\end{abstract}

\section{Introduction}

Scatter factor (SF) and HGF were discovered independently as a fibroblast-derived morphogen for epithelial cells and a mitogen for primary rat hepatocytes, respectively (1-4). Further biochemical characterization of the proteins, however, revealed that the two molecules are identical (5-7). It is now well established that $\mathrm{SF} / \mathrm{HGF}$ (referred to as SF here) is a pleiotropic factor capable of controlling the proliferation, motility, survival, and morphology of a wide spectrum of tissues, including cells of epithelial, endothelial, hemopoietic, and neuronal origin (8-10). The harmonic coordination of these biological activities by SF promotes a characteristic genetic program known as "invasive growth," which plays a central role in both physiological and pathological processes (reviewed in refs. 11 and 12).

In the developing embryo, SF gradients drive invasion of the uterine matrix by the trophoblast, neoangiogenesis in the developing organs, and migration of differentiating epithelial, neural, muscle, and hemopoietic precursor cells (13-16). Mice lacking the $s f$ gene, or lacking the gene encoding for the high-affinity receptor of $\mathrm{SF}$, the tyrosine kinase Met, die in utero due to placental and hepatic defects (16-18). In the adult organism, SF has been shown to participate in tissue regeneration (19), wound healing (20), and hematopoiesis (21), all processes that somehow recapitulate the invasive activity of a forming organ. This pro-invasive mimicry, however, is at its maximum in malignant tumors, in which the SF/Met pathway plays a crucial role in peri-

Nonstandard abbreviations used: poly-His, poly-histidine; SF, scatter factor; uPA, urokinase-type plasminogen activator.

Conflict of interest: The authors have declared that no conflict of interest exists.

Citation for this article: J. Clin. Invest. 114:1418-1432 (2004).

doi:10.1172/JCI200422235. tumoral matrix remodeling; cancer cell proliferation, migration, and survival; tumor angiogenesis; and finally metastatic colonization (reviewed in ref. 22).

$\mathrm{SF}$ is secreted in the form of a single-chain inactive precursor (pro-SF), which needs proteolytic cleavage in order to become biologically active $(23,24)$. Pro-SF is produced by cells of mesenchymal origin but it is abundant in the extracellular matrix of almost all tissues, where it is retained due to its high affinity for proteoglycans $(25,26)$. Picomolar concentrations of pro-SF are also present in plasma, where they often fluctuate in relation to particular physiological or pathological conditions (27-29). Proteolytic activation of pro-SF occurs locally and is typically carried out by proteases produced by the target tissue or by factors involved in blood clotting and fibrinolysis (30-33). As a consequence of proSF ubiquity, pro-factor conversion and receptor expression regulate SF activity in vivo.

There are several examples of this paradigm in both normal and tumor tissues. After tissue damage, upregulation of pro-SF convertases, including urokinase-type plasminogen activator (uPA) and HGF activator, catalyzes the conversion of locally stored proSF into active SF. Higher concentrations of active SF protect cells against apoptosis and promote tissue regeneration (30, 34-36). Invasive tumors may constitutively overexpress proteases involved in pro-SF conversion, thus exploiting the pro-SF reservoir more actively than normal tissues or their less invasive counterparts $(37,38)$. In poorly vascularized tumors or in rapidly growing lesions, where oxygen is limited, hypoxia induces transcription of the met gene. Higher levels of SF receptor render tumor cells more sensitive to environmental SF, thus activating an invasive program that leads them away from the hypoxic area (39). Interestingly, hypoxia also induces higher levels of the uPA receptor (40), which actively participates in pro-SF conversion (41). 
This in vivo regulatory mechanism offers at least 2 distinct opportunities for therapeutic intervention. The first would be interference with ligand-receptor interaction. A therapeutic molecule that impairs SF binding to Met would keep the receptor in an inactive state even if the tumor were rich in active SF. The second would be at the level of the conversion of pro-SF into active SF. If no active SF is available in the tumor microenvironment, tumor cells or tumor-infiltrated endothelial cells will not receive pro-invasive signals even if their Met levels ensure high ligand sensitivity. Although the former approach has already been attempted with some encouraging results (42-44), the latter strategy has not as yet been explored in preclinical settings.

In this study, we present a gene therapy approach that achieves both of the goals above via the delivery of a single molecule. By introducing a single-amino acid substitution in the proteolytic site, we generated an unprocessable form of pro-SF (uncleavable SF). We show that uncleavable SF conserves the ability to interact with proSF convertases and to bind the Met receptor with high affinity, thus inhibiting both endogenous pro-SF conversion and SF-induced receptor activation.

\section{Results}

Engineering of an unprocessable form of pro-SF. Pro-SF is converted to active SF by hydrolysis of the peptidic bond between Arg 489 and Val 490 (23). To generate an unprocessable form of pro-SF, we mutagenized Arg 489 to Gln, thus destroying the proteolytic consensus sequence Lys-Arg-Val-Val (Figure 1A). To allow simple protein purification, we also engineered a poly-histidine (polyHis) tag at the $\mathrm{C}$ terminus. The cDNA encoding the mutagenized protein (uncleavable SF) as well as cDNA encoding wild-type SF (engineered with an identical poly-His tag) were subcloned into the lentivirus vector pRRLsin.PPT.CMV.Wpre (45). Using these transgenic lentivirus vectors, we transduced MDA-MB-435 human mammary carcinoma cells and established 2 cell lines that secreted high levels of uncleavable SF or wild-type pro-SF into the culture medium (approximately 200 pmol per $10^{6}$ cells in 72 hours). Recombinant uncleavable SF and wild-type pro-SF were purified to homogeneity by affinity chromatography (Figure 1B) and then were subjected to proteolytic conversion assays.

We incubated a fixed amount of pro-SF or uncleavable SF with increasing concentrations of UPA or FBS, which contains several proteases capable of converting pro-SF into active SF (e.g., HGF activator and factors X, XI, and XII). After the reaction, the amount of active factor was assessed by Western blot with antibodies against the $\beta$-chain of SF. As shown in Figure 1C, purified uPA or proteases contained in serum completely converted wild-type pro-SF into active SF but failed to activate uncleavable SF, thus confirming that the mutagenized arginine is essential for proteolytic processing.

Uncleavable SF binds the Met receptor with high affinity. Although unable to induce signaling, pro-SF is known to bind the Met receptor with high affinity $(41,46)$. To test the ability of uncleavable SF to bind the Met receptor, we performed ELISA assays using purified Met and ligand-displacement assays using radiolabeled uncleavable SF. ELISA assays were performed using purified Met (44) in solid phase and increasing concentrations of wild-type pro-SF, active $\mathrm{SF}$, or uncleavable SF in liquid phase. This analysis revealed that uncleavable SF or pro-SF bound purified Met with approximately 4-fold less affinity than active SF (Figure $1 D$ ); i.e., with a $K_{\mathrm{d}}$ of approximately $0.2 \mathrm{nM}$. Ligand-displacement assays were conducted using various cell lines expressing Met (A549, human lung carci- noma; MDA-MB-435, human breast carcinoma; GTL-16, human gastric carcinoma). A fixed concentration of ${ }^{125}$ I-labeled uncleavable $\mathrm{SF}(0.25 \mathrm{nM})$ was displaced with increasing concentrations of unlabeled wild-type pro-SF, active SF, or uncleavable SF. In all cell lines tested, all factors achieved complete displacement of ${ }^{125} \mathrm{I}$-labeled uncleavable SF, although with different efficiency. The calculated $50 \%$ effective concentration for active SF was 4-6 times lower than that for pro-SF or uncleavable SF, depending on the cell line (A549, 5.5 times; MDA-MB-435, 6 times; GTL-16, 4 times). Figure 1E shows displacement data obtained in A549 cells. These results are consistent with previous measurements (46).

Uncleavable SF inhibits SF-induced Met activation. We next tested the ability of uncleavable SF to activate the Met receptor in tyrosine phosphorylation assays. Serum-starved A549 human lung carcinoma cells were stimulated with increasing concentrations of active SF, pro-SF, or uncleavable SF, and the extent of Met phosphorylation was assessed by Western blot using anti-phosphotyrosine. As shown in Figure 2A, active SF elicited dose-dependent phosphorylation of Met, while both pro-SF and uncleavable SF were completely inactive even at high doses. Therefore, uncleavable SF is a mutant form of pro-SF that cannot be converted to active SF by proteolysis, binds to Met with picomolar affinity, and is not capable of activating the Met receptor in tyrosine phosphorylation assays.

Based on these observations, we tested whether uncleavable SF could work as a competitive inhibitor of SF in Met activation experiments. We stimulated A549 cells with a fixed amount of active $\mathrm{SF}(0.44 \mathrm{nM})$ plus increasing concentrations of uncleavable SF or pro-SF as a control, and then assessed Met phosphorylation as described above. This analysis revealed that both pro-SF and uncleavable SF are powerful antagonists of SF. In fact, a significant inhibition of receptor phosphorylation was observed with only a 6-fold molar excess of pro-SF or uncleavable SF, and 40-fold molar excess achieved complete inhibition (Figure 2B).

Uncleavable SF inhibits pro-SF conversion by $\mathrm{UPA}$ and other proteases. In principle, uncleavable SF is indistinguishable from pro-SF, at least from a structural viewpoint. We therefore reasoned that in addition to displacing the ligand from the receptor, uncleavable SF could also compete with wild-type pro-SF for the active site of uPA or other proteases involved in pro-SF conversion. To test this hypothesis, we incubated a fixed amount of Myc epitope-tagged pro-SF with either purified uPA or FBS, plus increasing concentrations of recombinant uncleavable SF. After the reaction, pro-SF conversion was assessed by Western blot with anti-Myc. In these assays, uncleavable SF inhibited proteolytic conversion of pro-SF in a dose-dependent manner. Equal concentrations of pro-SF and uncleavable SF resulted in approximately $50 \%$ inhibition of pro-SF conversion, while nearly complete inhibition was observed with a 16 -fold molar excess (Figure 2C).

Uncleavable SF forms stable complexes with Met and uPA in living cells. Prompted by the results above, we investigated whether uncleavable SF could form stable complexes with Met or uPA in living cells. To this end, we cross-linked ${ }^{125}$ I-labeled uncleavable SF to A549 cells and then immunoprecipitated cell lysates with anti-Met or with anti-uPA. Immunoprecipitated proteins were resolved by gradient SDS-PAGE in nonreducing conditions and were visualized by autoradiography. As shown in Figure 2D, anti-Met immunoprecipitated radiolabeled high-molecular-weight complexes (molecular weight of more than $206 \mathrm{kDa}$ ), suggesting that uncleavable SF and Met interact stably on the cell surface. Anti-uPA immunoprecipitated radiolabeled species of between 120 and $140 \mathrm{kDa}$, corresponding to a complex of uPA 
A

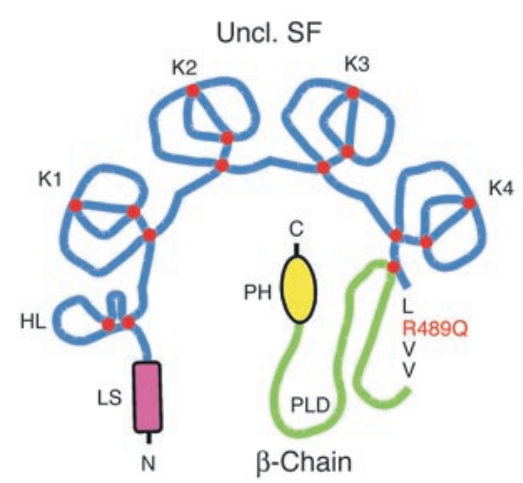

B

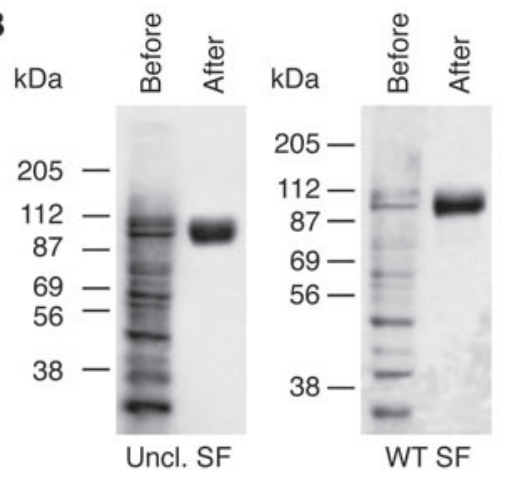

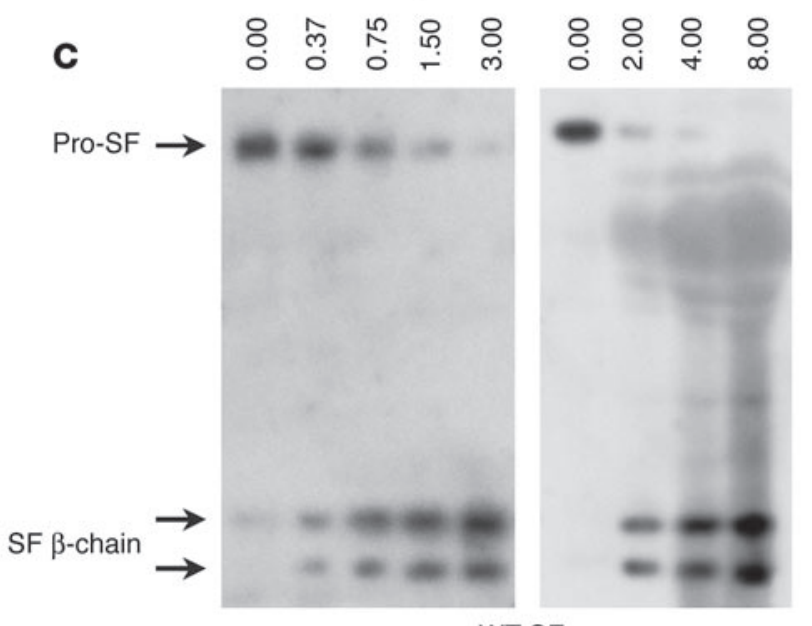

WT SF

D

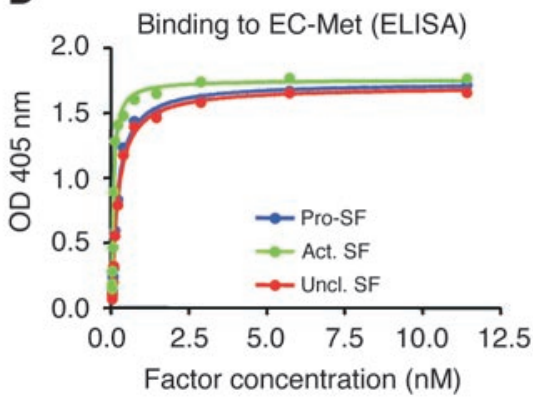

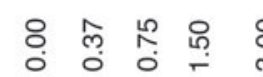
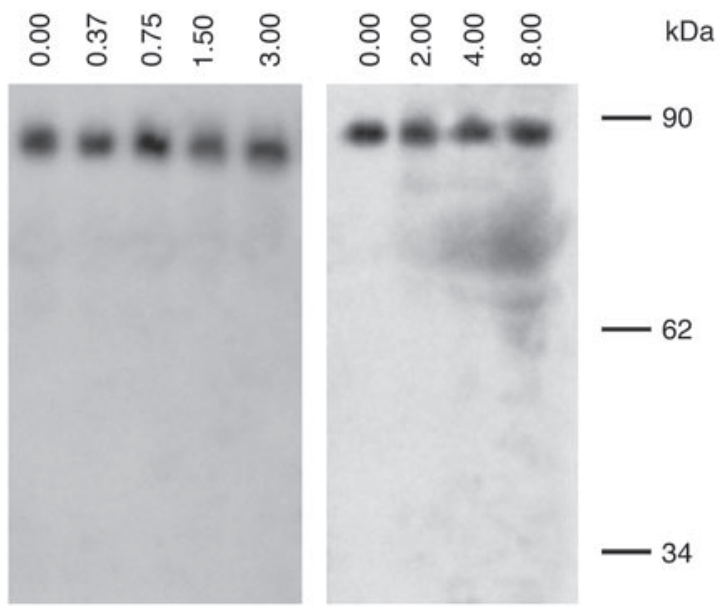

Uncl. SF
E Displacement of ${ }^{125}$-labeled uncl. SF (A549 cells)

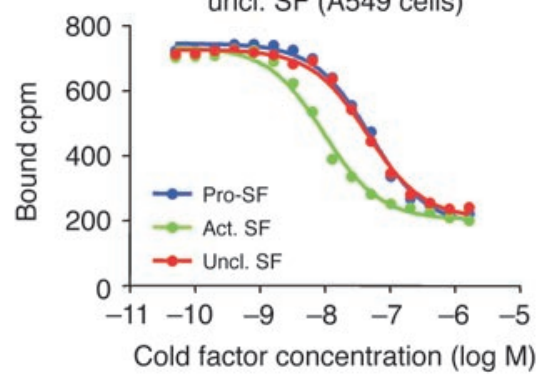

\section{Figure 1}

Uncleavable SF is resistant to proteolytic activation, but binds Met with high affinity. (A) Schematic representation of uncleavable SF (Uncl. SF; from $\mathrm{N}$ to $\mathrm{C}$ terminus): LS, leader sequence; HL, hairpin loop; K1-K4, kringle 1-4; PLD, protease-like domain; PH, poly-His. The Arg-toGIn substitution in the proteolytic site is shown in red. Red dots indicate disulfide bridges. (B) Purification of wild-type or uncleavable SF by affinity chromatography, before and after purification. Proteins were resolved by nonreducing SDS-PAGE. (C) Uncleavable SF is resistant to proteolytic conversion by UPA and FBS. Proteins were resolved by SDS-PAGE in reducing conditions and analyzed by Western blot using antibodies against the $\beta$-chain of SF. The 2 SF $\beta$-chain bands correspond to differently glycosylated forms. (D) Binding of uncleavable SF to purified extracellular Met (EC-Met) as assessed by ELISA. Bound SF was revealed using biotinylated anti-SF. (E) Binding of iodinated uncleavable SF to A549 human lung carcinoma cells. ${ }^{125}$ I-labeled uncleavable SF was displaced using increasing concentrations of cold pro-SF, active SF (Act. SF), or uncleavable SF.

in either of its forms ( 33 and $54 \mathrm{kDa})$ with pro-SF $(90 \mathrm{kDa})$. Covalent cross-linking of ${ }^{125}$ I-labeled uncleavable SF to Met or uPA was efficiently displaced by excess unlabeled uncleavable SF, wild-type pro-SF, or active SF, indicating that the interaction of uncleavable SF with Met or uPA is specific and demonstrating that uncleavable SF and wild-type SF (in either of its forms) compete for the same molecular partners in living cells.
Delivery of uncleavable SF to tumor cells by lentivirus vector technology. Using the lentivirus vectors described above and an empty vector as control, we transduced a panel of Met-expressing human tumor cell lines (MDA-MB-435 and MDA-MB-435- $\beta 4$, mammary carcinoma; A549, lung carcinoma; HCT-116, colon carcinoma). Factor expression in the presence or absence of serum was analyzed by Western blot using antibodies against the $\beta$-chain of SF. As shown 
A

A
IP anti-Met
WB anti-pY
IP anti-Met
WB anti-Met

Active SF (nM)

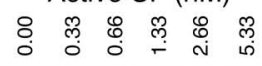

Pro-SF (nM)

Uncl. SF (nM)

\&
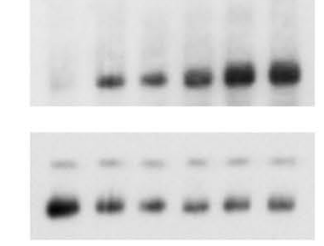
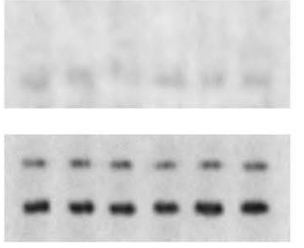
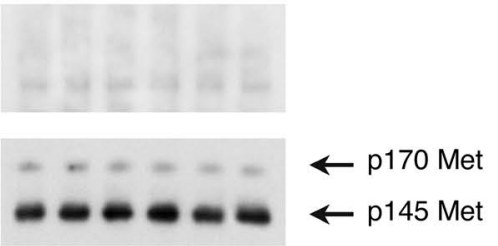

B

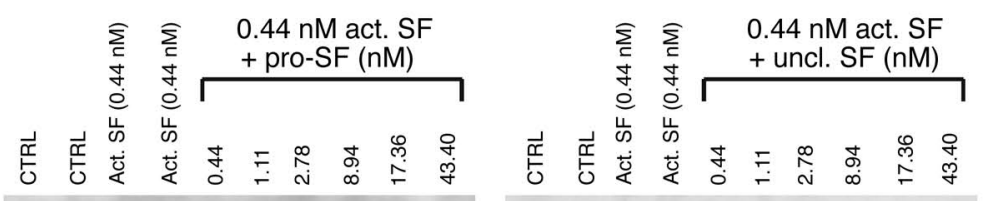

IP anti-Met

WB anti-pY
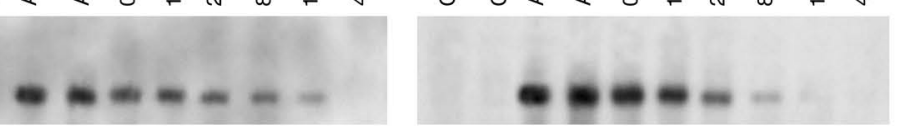

IP anti-Met
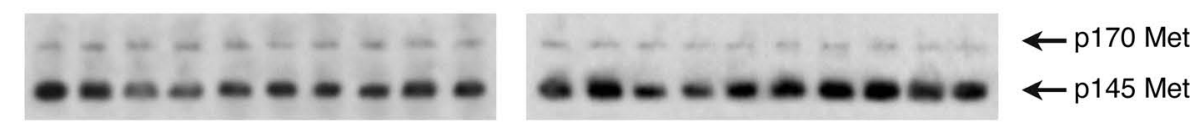

C

11.11 nM Myc-tagged pro-SF + uncl. SF (nM)
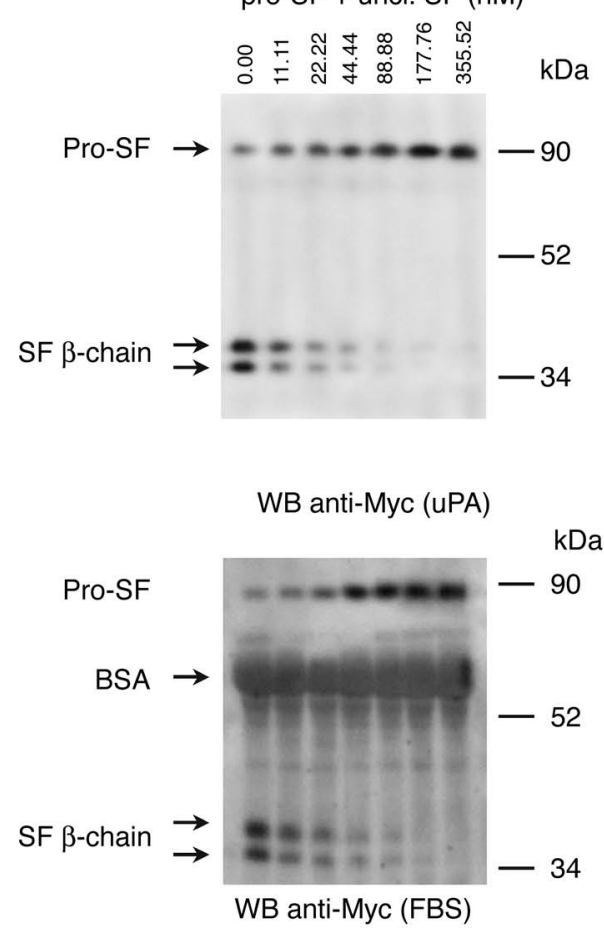

D
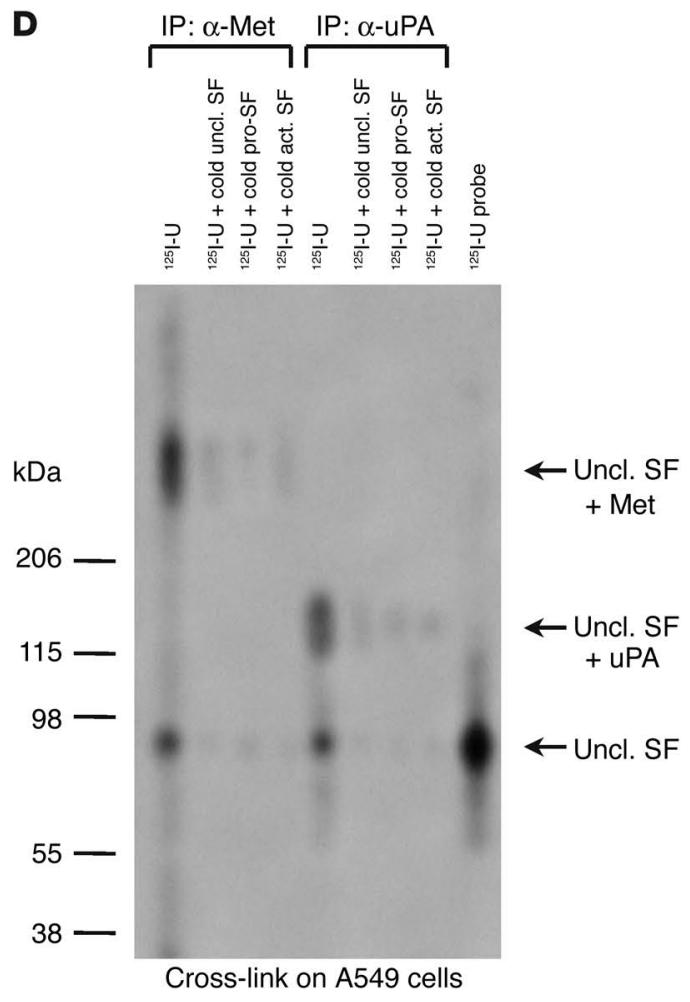

\section{Figure 2}

Uncleavable SF inhibits both SF-induced Met activation and protease-mediated pro-SF conversion. (A) Met activation assay. A549 cells were stimulated with active SF, pro-SF, or uncleavable SF, and the extent of Met phosphorylation was assessed using anti-phosphotyrosine (anti-pY). The 2 receptor bands correspond to unprocessed Met ( $p 170$, in the Golgi) and mature Met ( $p 145$, on the cell membrane). The p170/p145 ratio varies depending on the cell line. IP, immunoprecipitation; WB, Western blot. (B) Inhibition of Met phosphorylation. Cells were stimulated with a fixed amount of active SF plus increasing concentrations of pro-SF (left panel) or uncleavable SF (right panel). CTRL, control (unstimulated cells). (C) Inhibition of pro-SF conversion. Myc-tagged pro-SF was incubated with a fixed amount of uPA (upper panel) or FBS (lower panel), plus increasing concentrations of uncleavable SF (above lanes). Pro-SF conversion was assessed by Western blot with anti-Myc. (D) Uncleavable SF forms stable complexes with Met and uPA. A549 cells were exposed to ${ }^{125}$ I-labeled uncleavable SF (125I-U) in the presence of a cross-linking agent and then were lysed. Protein extracts were immunoprecipitated using anti-Met or anti-uPA, resolved by SDS-PAGE in nonreducing conditions, and visualized by autoradiography. 


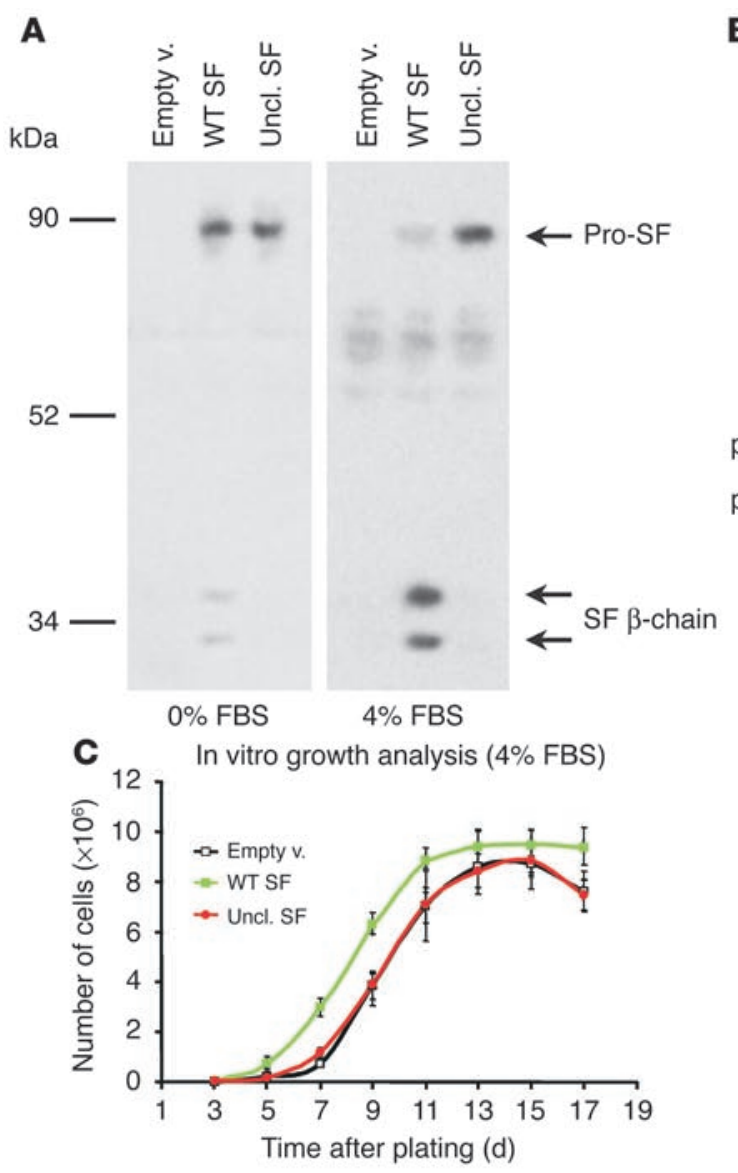

B
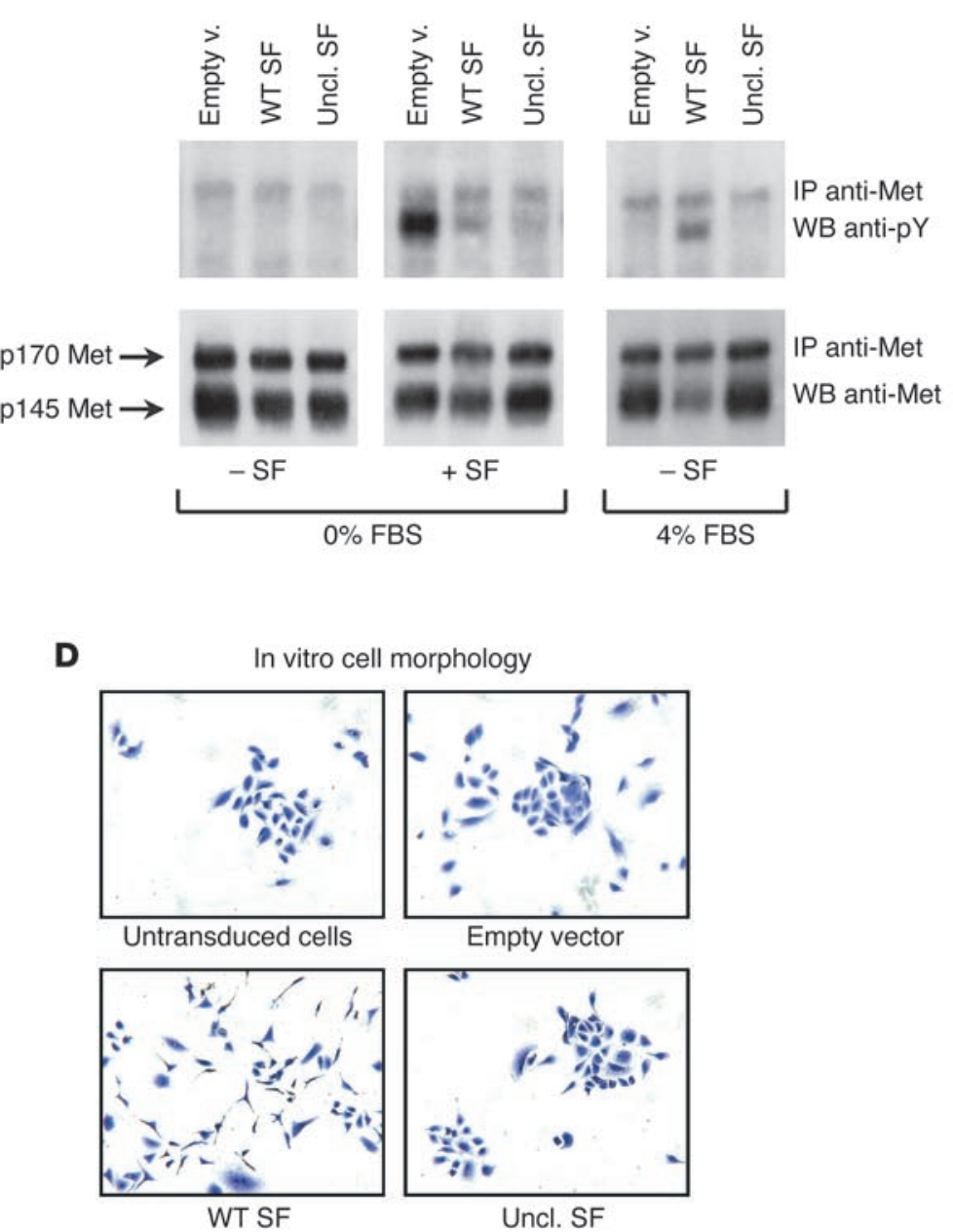

\section{Figure 3}

Delivery of uncleavable SF to tumor cells using lentivirus vectors. (A) MDA-MB-435 cells transduced with the indicated lentivirus vector (empty vector [Empty v.]; uncleavable SF; WT SF) were cultured in the absence (0\%) or presence (4\%) of FBS, and their conditioned medium was analyzed by Western blot using anti-SF $\beta$-chain. (B) Analysis of Met phosphorylation in lentivirus vector-transduced MDA-MB-435 cells. Receptor activation was assessed by Western blot using anti-phosphotyrosine in quiescent ( $0 \%$ FBS) or exponentially growing (4\% FBS) cells in the presence (+) or absence (-) of recombinant SF, as indicated. (C) Proliferation rate analysis of lentivirus vector-transduced MDA-MB-435 cells. Cell numbers were assessed every 2 days. (D) In vitro morphology of lentivirus vector-transduced A549 cells stained with crystal violet.

in Figure 3A (only the data relative to MDA-MB-435 are presented), wild-type SF could be detected in its active form only in the presence of serum. Serum-free conditioned media contained only traces of active SF, possibly due to endogenous protease activity. In contrast, uncleavable SF was detected as an unprocessed protein regardless of the presence of serum.

Transduced MDA-MB-435 cells were subjected to a Met phosphorylation assay using exogenous recombinant SF. In a first set of experiments, cells were stimulated with SF in the absence of serum. Met phosphorylation was assessed by Western blot with anti-phosphotyrosine. Consistent with the idea that pro-SF competes with active SF in Met binding, both uncleavable SF and wildtype SF inhibited SF-induced Met phosphorylation in the absence of serum (Figure 3B, $0 \%$ FBS panel). In a second set of experiments, Met phosphorylation was assessed in exponentially growing cells, in both the presence of serum and the absence of exogenous SF
(Figure 3B, 4\% FBS panel). In these conditions, cells expressing wild-type SF displayed intense Met phosphorylation and receptor downregulation. In contrast, cells expressing uncleavable SF showed no significant change in Met phosphorylation or receptor levels compared with that of the control.

In long-term proliferation assays in the presence of 4\% FBS, all cells expressing wild-type SF grew slightly faster than control cells and reached a higher saturation density (Figure 3C; only the data relative to MDA-MB-435 are shown). In contrast, expression of uncleavable SF did not significantly affect cell growth or saturation density, suggesting that the engineered factor does not display cellular toxicity (none of the cell lines analyzed express endogenous SF). Most cells transduced with SF lentivirus also displayed a more scattered phenotype. This phenomenon was best observed in A549 (Figure 3D). Cells transduced with uncleavable SF lentivirus, in contrast, showed a morphology indistinguishable from that of control. 
A

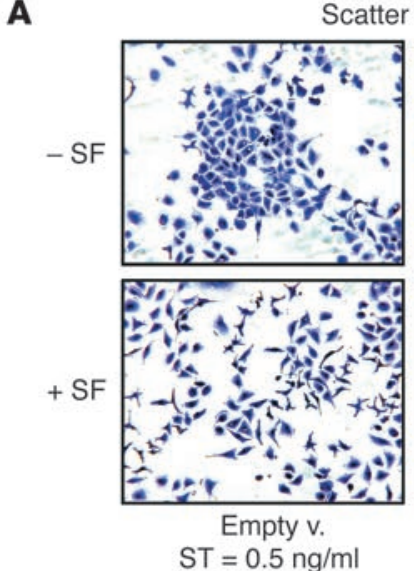

C

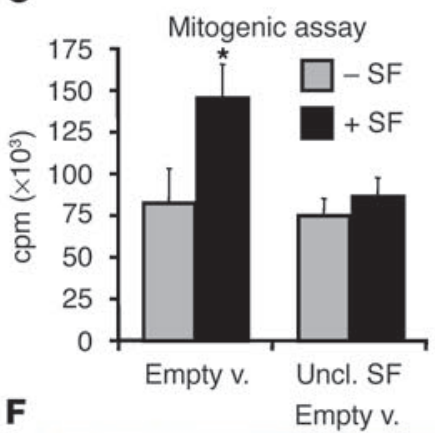

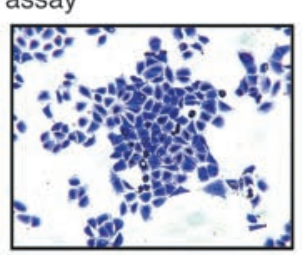

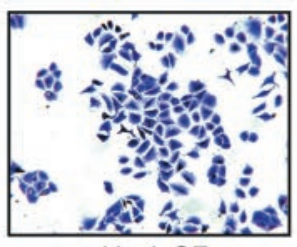

Uncl. SF

$\mathrm{ST}=16 \mathrm{ng} / \mathrm{ml}$

D

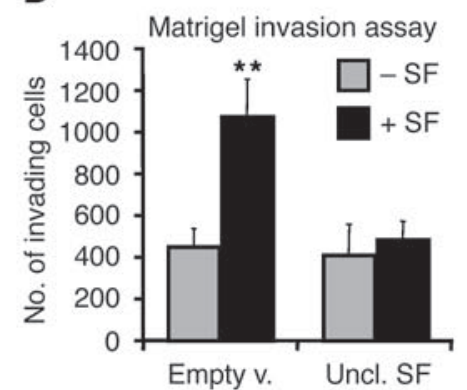

Survival assay

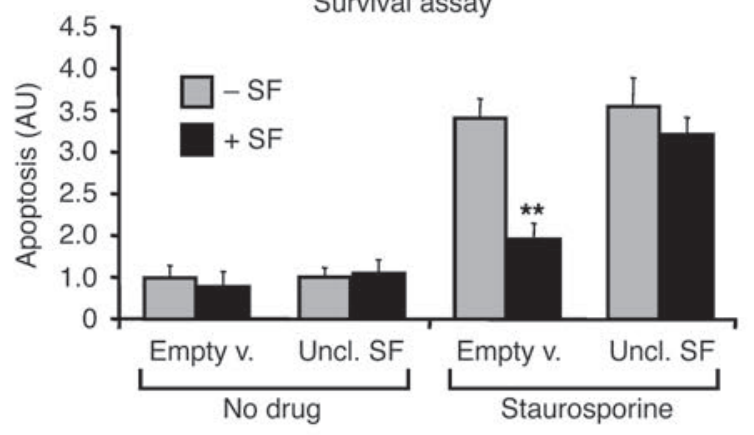

E

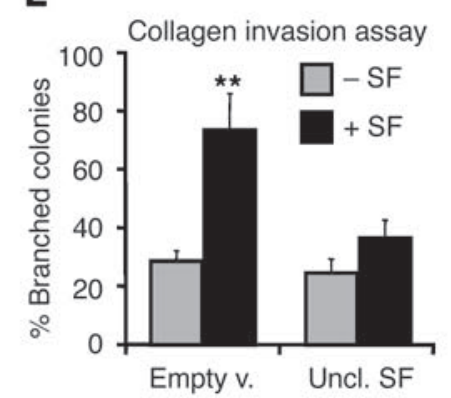

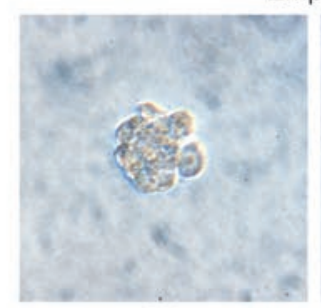

$-\mathrm{SF}$

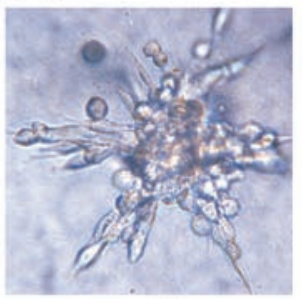

+ SF

Uncl. SF

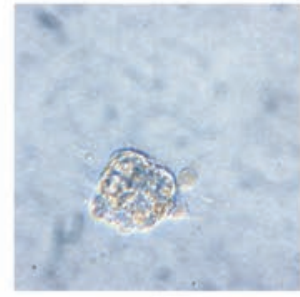

-SF

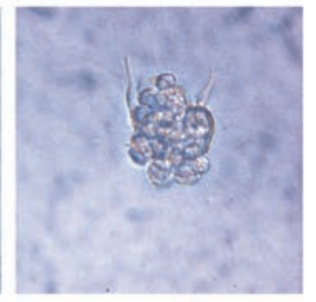

+ SF

Figure 4

Uncleavable SF inhibits the pleiotropic activities of SF on living cells. (A) End-point scatter assay. A549 cells transduced with the indicated lentivirus vector were stimulated with progressive $1: 2$ dilutions of active SF starting from $64 \mathrm{ng} / \mathrm{ml}$. The minimal concentration at which scattering was observed is indicated (scatter threshold [ST]). Representative images show crystal violet-stained cells at $8 \mathrm{ng} / \mathrm{ml}$ (the concentration of active SF). (B) Survival assay. Lentivirus vector-transduced MDA-MB-435 cells were pre-incubated with either recombinant SF (+SF) or no factor (-SF) and then were cultured in the absence (No drug) or presence (Staurosporine) of staurosporine. (C) Mitogenic assay. Lentivirus vector-transduced A549 cells were stimulated with either recombinant SF or no factor, and DNA synthesis was assessed by [ $\left.{ }^{3} \mathrm{H}\right]$ thymidine incorporation. (D) Matrigel invasion assay. MDA-MB-435- $\beta 4$ cells were analyzed for their ability to invade a Matrigel layer in the presence or absence of recombinant SF. (E) Collagen invasion assay. Lentivirus vector-transduced MDA-MB-435 cells were examined for their ability to form branched, multicellular tubules in response to SF stimulation. (F) Representative images from the experiment described in E. Statistical significance $\left({ }^{\star} P<0.05 ;{ }^{*} P<0.01\right)$ refers to the difference between $-\mathrm{SF}$ and $+\mathrm{SF}$.

Uncleavable SF inhibits the pleiotropic activities of SF in vitro. The ability of uncleavable SF to inhibit the pleiotropic activities of SF was analyzed in a series of in vitro biological assays. Lentivirus vectortransduced cells, selected based on assay suitability and response to SF, were subjected to a "scatter" assay (A549), a survival assay (MDA-MB-435), a mitogenic assay (A549), a Matrigel invasion assay (MDA-MB-435- $\beta 4$ ), and a branching morphogenesis assay (MDA-MB-435- $\beta 4$ ), in the absence or presence of recombinant SF. This analysis revealed that the concentrations of uncleavable SF reached in the conditioned medium of transduced cells (20-100 $\mathrm{nM}$ depending on cell line and incubation time) effectively inhib- ited SF-induced cell scattering (Figure 4A), protection against apoptosis (Figure 4B), DNA synthesis (Figure 4C), Matrigel invasion (Figure 4D), and branching morphogenesis (Figure 4E), thus demonstrating that the engineered factor can functionally neutralize the effects of SF on living cells.

Local expression of uncleavable SF prevents tumor growth and dissemination. We next analyzed the biological activity of wild-type SF and uncleavable SF in ex vivo tumorigenesis assays in mice. The full panel of lentivirus vector-transduced cell lines (MDA-MB-435, MDA-MB-435- $\beta 4$, A549, and HCT-116; each line was transduced with empty lentivirus vector, wild-type SF lentivirus vector, or 
A

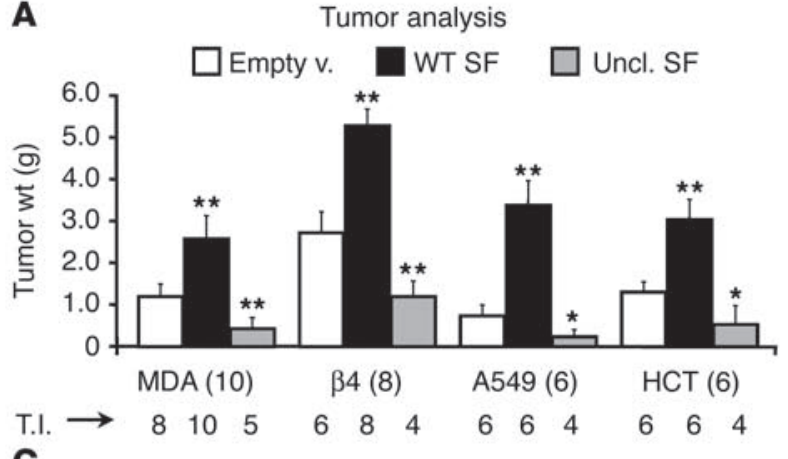

B

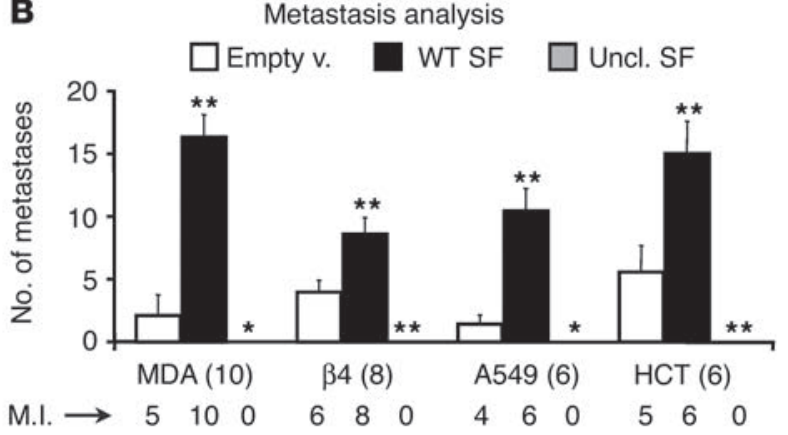

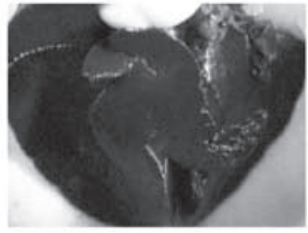

Normal lungs

D

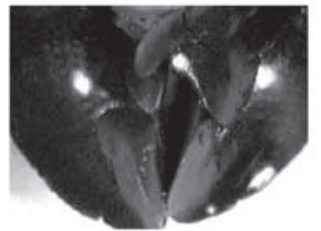

Empty v.

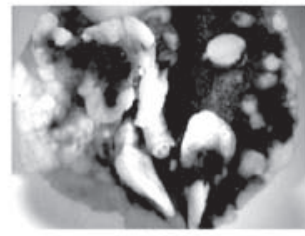

WT SF

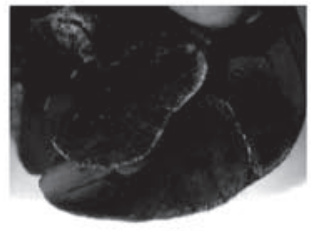

Uncl. SF

E Experimental metastases

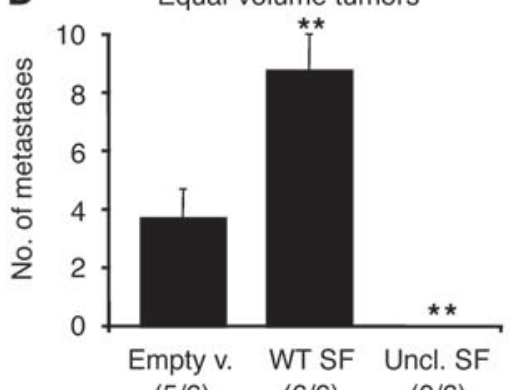

$(5 / 6)$

$(6 / 6)$

(0/6)

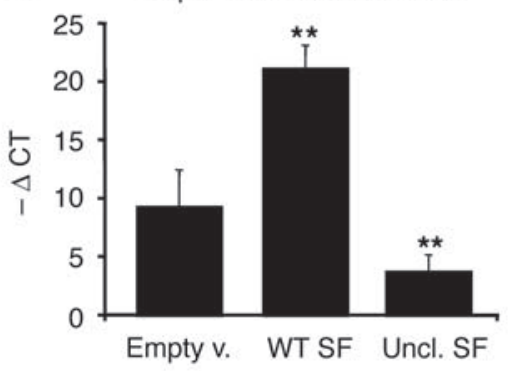

$\mathbf{F}$

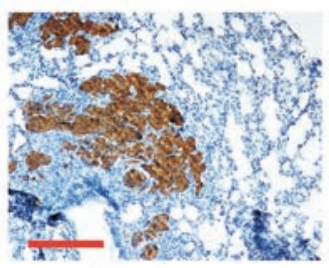

Empty v.

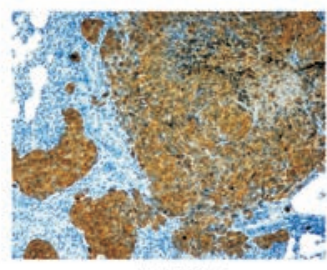

WT SF

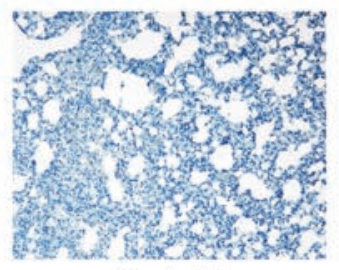

Uncl. SF

Figure 5

Uncleavable SF inhibits tumor growth and metastasis in mice. (A) Ex vivo tumorigenesis assay. A panel of human tumor cell lines (MDA, MDAMB-435; $\beta 4$, MDA-MB-435- $\beta 4$; A549; HCT, HCT-116) transduced with the indicated lentivirus vectors was injected subcutaneously into nude mice and tumor weight was determined after 50 days. Numbers in parentheses indicate the number of mice per group. T.l., tumor incidence (number of mice with tumor). (B) Analysis of pulmonary metastases in mice described in A. M.l., metastasis incidence (number of mice with metastases). Cells expressing uncl. SF were not metastogenic. (C) Representative images from the analysis described in B (MDA-MB-435). Lungs were stained with black India ink through the trachea. Metastases appear white because they do not take up the dye. (D) Metastasis analysis in mice bearing tumors of equal size. The number of pulmonary metastases was determined as described in $\mathbf{B}$ when tumors reached a volume of $3,000 \mathrm{~mm}^{2}$. Metastatic incidence is shown in parenthesis. (E) Experimental metastasis assay. HCT-116 cells engineered to express GFP were transduced with the indicated lentivirus vector and were injected i.v. into nude mice. The extent of pulmonary colonization was assessed by TaqMan analysis of lung genomic DNA using a gfp-specific probe. Quantification is expressed as cycle threshold difference compared with negative control $(-\Delta C T)$. (F) Representative lung sections from the experiment described in E, stained with anti-GFP. Red scale bar: $500 \mu \mathrm{m}$. Statistical significance $\left({ }^{\star} P<0.05 ;{ }^{* \star} P<0.01\right.$ ) was calculated by comparison of experimental values (WT SF or Uncl. SF) with control values (Empty v.).

uncleavable SF lentivirus vector, thus generating 12 different transgenic cell lines) was injected subcutaneously into CD-1 $\mathrm{nu}^{-/-}$ mice, and injected animals were monitored for tumor development. Each transgenic cell line was injected into 6 mice, for a total of 72 mice; all mice were injected at the same time. After approximately 50 days, experimental tumors were extracted for analysis and India ink was used to provide contrast between metastic nodules and normal tissue. After scores for metastasis were assigned, lungs were processed for histological analysis.

Remarkably, wild-type SF and uncleavable SF (differing by only 1 amino acid) elicited symmetrical effects on both tumor growth and dissemination in all cell lines tested (Figure 5A). Wild-type SF 


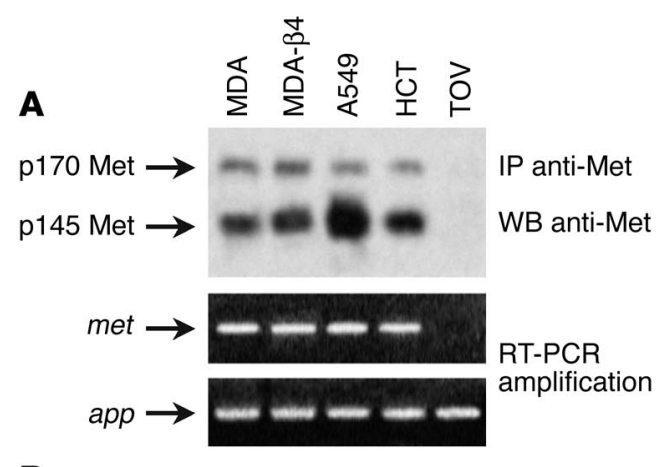

B

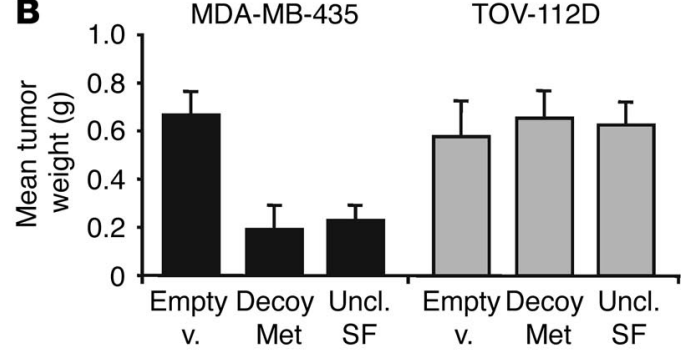

Figure 6

Uncleavable SF does not affect the growth of tumors lacking Met. (A) Analysis of Met protein and met mRNA expression in human tumor cell lines used in this study. MDA- $\beta 4$, MDA-MB-435- $\beta 4$; TOV, TOV- $112 \mathrm{D}$; app, human housekeeping gene encoding $\beta$-amyloid precursor protein (used as positive control for PCR amplification). (B) Ex vivo tumorigenesis assay. Lentivirus vector-transduced cells were injected into nude mice as described above and tumor weight was determined after 1 month. Statistical significance was calculated as for Figure 5. Decoy Met is a soluble form of the Met receptor (see ref. 44).

accelerated tumor xenograft implantation and augmented tumor incidence, increased tumor weight ( 2 - to 5 -fold; $P<0.010$ ), and enhanced the number of metastases ( 2 - to 8-fold; $P<0.010)$. In contrast, uncleavable SF increased both tumor latency and the number of tumor-free animals, reduced tumor weight (2- to 3-fold; $P<0.050$ ), and completely prevented the formation of spontaneous metastases (see Figure 5C for representative images). Mice injected with cells transduced with uncleavable SF lentivirus were also free of micrometastases, as assessed by histological analysis of lung sections (not shown).

To determine whether these changes in metastatic incidence were merely due to increased or reduced primary tumor size, we performed the same metastasis analysis on a panel of animals bearing MDA-MB- $435-\beta 4$ tumors of the same volume (approximately $3,000 \mathrm{~mm}^{3}$ ) at the time of sacrifice, regardless of the time required by the tumor to reach such volume (Figure 5D). In this analysis as well, no metastasis could be found in the group transduced with uncleavable SF lentivirus, while wild-type SF considerably increased the number of metastases compared with empty vector. Thus, uncleavable SF inhibits tumor dissemination independently of its parallel action on tumor growth.

The antimetastatic effect of uncleavable SF was further examined in ex vivo pulmonary colonization assays. HCT-116 tumor cells were transduced with a lentivirus encoding GFP and the various lentivirus vector constructs described above. Transduced cells were injected i.v. into CD-1 $n u^{-/-}$mice, and GFP-positive pulmonary colonies (experimental metastases) were analyzed
1 month later by TaqMan analysis of lung genomic DNA and immunohistochemistry of lung sections with anti-GFP. While wild-type SF considerably increased the number of GFP-positive cells in the lung and the extension of the metastatic lesions, uncleavable SF nearly completely abolished the survival and spreading of injected tumor cells (Figure 5, D and E).

Uncleavable SF does not affect the growth of tumors lacking Met. The data above suggest that uncleavable SF significantly inhibits the growth and dissemination of a variety of tumor xenografts. In our in vitro biochemical analysis, we have shown that uncleavable SF inhibits both SF-induced Met activation and protease-mediated pro-SF conversion (see Figure 2). Because some of the proteases involved in pro-SF conversion, in particular uPA, play an important role in tumor progression (37), we wondered whether the observed anti-tumoral activity could be attributed, in addition to impairment of HGF/Met signaling, to protease inhibition. To address this question, we performed a tumorigenesis assay using tumor cells lacking the Met receptor. We reasoned that if uncleavable SF exerted its anti-tumor activity through inhibition of pathways other than that of SF/Met, it would have also been effective against tumors that do not express Met. It is true that SF is involved in control of host angiogenesis as well, but we demonstrated previously that the anti-angiogenic effect of SF inhibitors itself is not sufficient to achieve permanent inhibition of tumor growth without a concomitant effect on tumor cells (44).

The human ovarian carcinoma cell line TOV-112D does not express Met, as assessed by both Western blot and RT-PCR (Figure $6 \mathrm{~A})$. TOV-112D cells were transduced with a lentivirus vector expressing uncleavable SF, an empty lentivirus vector as negative control, and a lentivirus vector expressing a "pure" HGF/Met antagonist as a positive control (decoy Met; ref. 44). Transduced cells were injected subcutaneously into nude mice as described above. As a reference, the same assay was conducted in parallel using a Met-positive cell line (MDA-MB-435). After 1 month, tumors were extracted and weighed. Because TOV-112D cells are not metastogenic in mice (44), tumor dissemination could not be analyzed. As shown in Figure 6B, both decoy Met (3.5-fold; $P=0.003)$ and uncleavable SF (2.9-fold; $P=0.005$ ) inhibited the growth of MDA-MB-435 cells in vivo, but neither significantly affected the formation of TOV-112D tumors (decoy Met, $P=0.375$; uncleavable $\mathrm{SF}, P=0.265$ ). These data suggest that Met-expressing tumor cells represent the major target of uncleavable SF in vivo, ruling out the possibility of a Met-independent mechanism of tumor inhibition.

Uncleavable SF arrests the progression of established tumors. Next, we analyzed the effect of uncleavable SF on the growth of established tumors. To this end, CD-1 $n u^{-/-}$mice were injected subcutaneously with MDA-MB-435- $\beta 4$ human mammary carcinoma cells and then were monitored for tumor appearance. After 2 weeks, all mice bearing tumors of approximately $100 \mathrm{~mm}^{3}$ were selected and randomly divided into three groups. High-titer lentivirus vector preparations were injected intratumorally and then tumor volume was measured every third day. After 18 days, tumors were extracted for analysis and mice were subjected to autopsy.

During the course of the experiment, tumors injected with empty vector enlarged by approximately 7 -fold, while those transduced with wild-type SF lentivirus expanded their volume by 12 -fold $(P=0.023)$. In contrast, uncleavable SF almost completely abolished the growth of injected tumors $(P=0.006$; Figure 7A). Similar results emerged from analysis of tumor weight (Figure 7B). Representative images of tumors can be seen in Figure 7C. Lungs were 
A

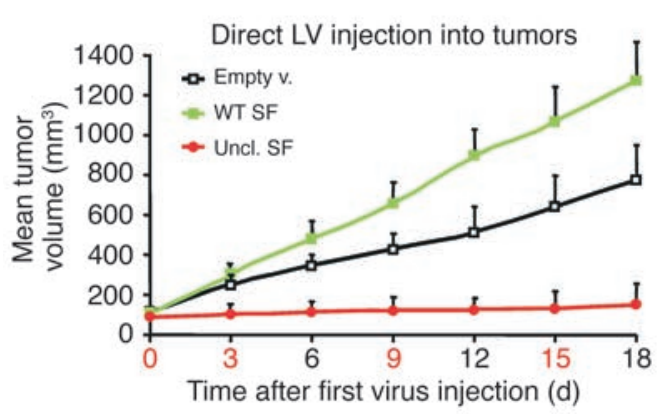

B

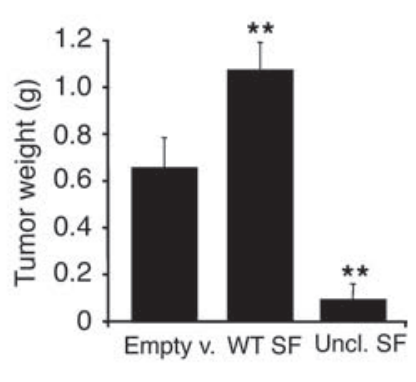

C

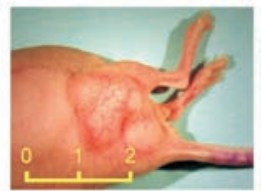

Empty v.

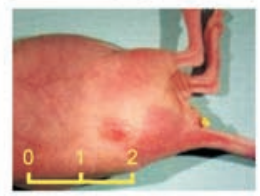

Uncl. SF
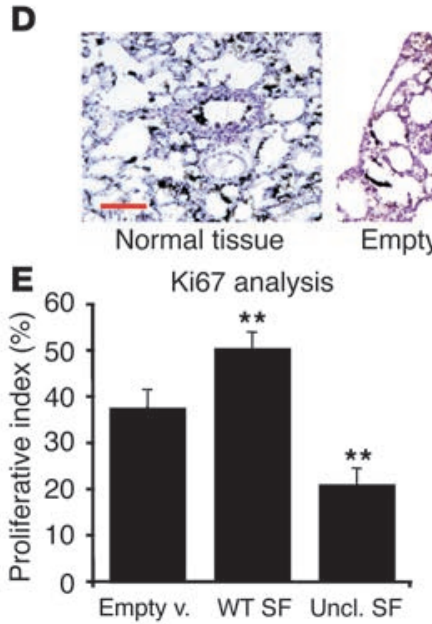

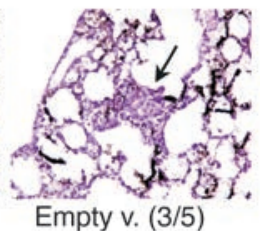

F

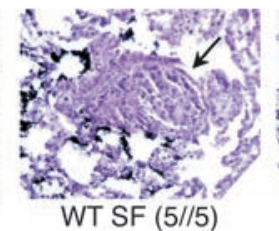

TUNEL analysis
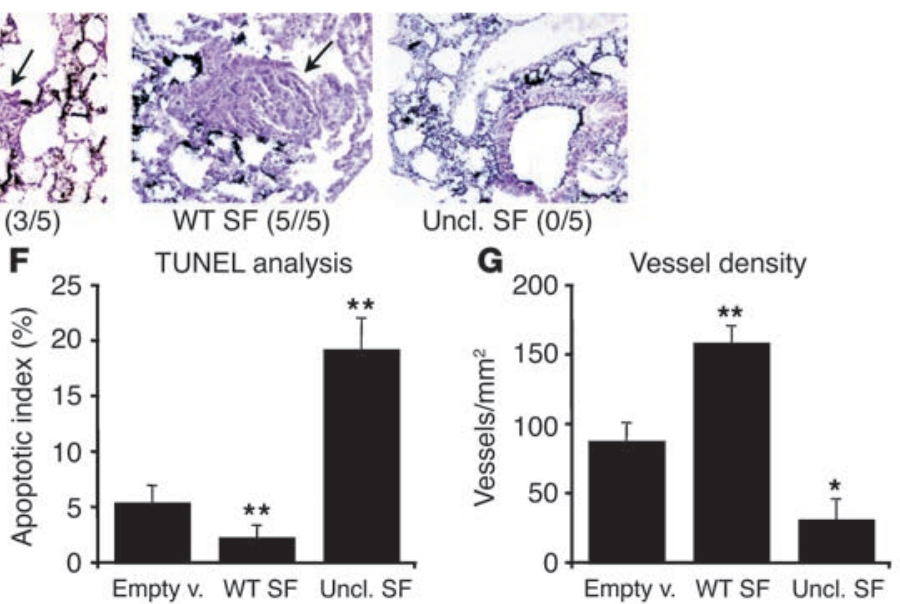

Figure 7

Uncleavable SF arrests the progression of established tumors. (A) Tumor burden analysis. Tumors of the same size (approximately $100 \mathrm{~mm}^{3}$ at day 0 ) were repeatedly injected with the indicated lentivirus vectors, and tumor volume was measured every 3 days. The numbers in red correspond to the days of lentivirus injection. LV, lentivirus vector. (B) Tumor weight analysis for the experiment described in $\mathbf{A}$. (C) Representative images of experimental tumors. The yellow ruler indicates length in centimeters. (D) Histological analysis of pulmonary metastases on lung sections stained with hematoxylin and eosin. A representative microscopic field for each group is shown. Arrows indicate the position of the metastatic lesion. Black staining is due to India ink. Metastasis incidence is shown in parenthesis. Red scale bar: $100 \mu \mathrm{m}$. (E) Tumor proliferation index analysis (\% Ki67-positive cells). (F) Tumor apoptotic index analysis (\% TUNEL-positive cells). (G) Tumor vessel analysis using the CD31 endothelial marker. Vessel density, number of vessels $/ \mathrm{mm}^{2}$. Statistical significance was calculated as for Figures 5 and 6.

contrasted with India ink as described above for metastasis analysis. No macrometastasis could be detected in any of the groups, possibly because a shorter incubation period was used here than in the ex vivo experiments. However, histological analysis revealed the presence of several micrometastases in mice injected with cells transduced with empty or SF vectors, but not in mice injected with cells transduced with uncleavable SF vector (Figure 7D). Metastatic lesions in the control group were sparse, small, and mostly parenchymatic; metastases of the group transduced with SF lentivirus were more frequent, larger, and typically embolic, with many "break" points in which cancer cells were invading the host parenchyma or interstitial space.

By analyzing frozen tumor sections, we also determined the proliferative index (Ki67; Figure 7E) and apoptotic index (TUNEL; Figure $7 F)$ of cells within the tumors. Tumors transduced with SF vector had a 1.3-fold higher proliferative index $(P=0.008)$ and a 2.4 -fold lower apoptotic index $(P=0.003)$ than those of control tumors. Conversely, tumor injection with uncleavable SF vector decreased cell proliferation by 1.8 -fold $(P<0.001)$ and augmented apoptosis by 3.6 -fold $(P=0.007)$. Because $\mathrm{SF}$ is a potent angiogenic factor $(47)$, we also analyzed tumor vessel density using the CD31 endothelial marker. As shown in Figure 7G, wild-type SF increased the number of vessels per surface unit by almost 2 -fold $(P<0.001)$, while uncleavable SF reduced it by approximately 3 -fold $(P=0.033)$.

Local expression of uncleavable SF prevents pro-SF conversion and inbibits Met phosphorylation. The same tumors described above were also analyzed for transgene expression, endogenous SF activation, and Met phosphorylation. To determine transgene expression, equal amounts of protein extracts from each tumor were immunoprecipitated with anti-pan SF, and were analyzed by Western blot with anti-human SF. As shown in Figure 8A (upper panel), similar amounts of wildtype SF or uncleavable SF could be detected in all tumors. Importantly, wild-type SF was present almost completely in its active form, suggesting that pro-SF convertases contained in the tumor were actively processing exogenous $\mathrm{SF}$.

Endogenous SF activation was assessed by immunoprecipitation with anti-pan SF and Western blot analysis with anti-mouse SF (Figure 8A, middle panel). Endogenous SF in tumors injected with empty vector was present mostly in the active form, although some precursor could be detected as well. Ectopic expression of uncleavable SF significantly shifted this balance toward the inactive form, thus suggesting that the engineered 
A

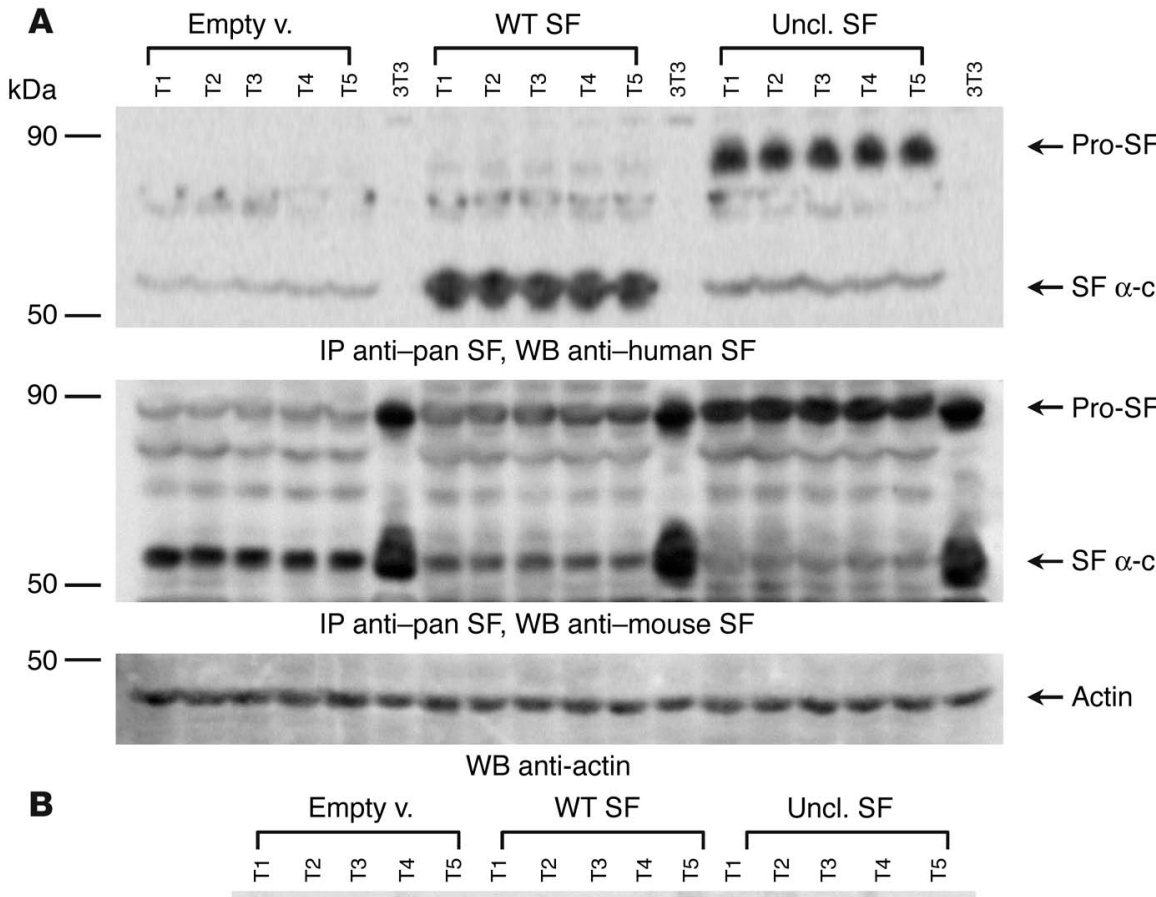

\section{IP anti-Met}

WB anti-pY

IP anti-Met

WB anti-Met

\section{wa}
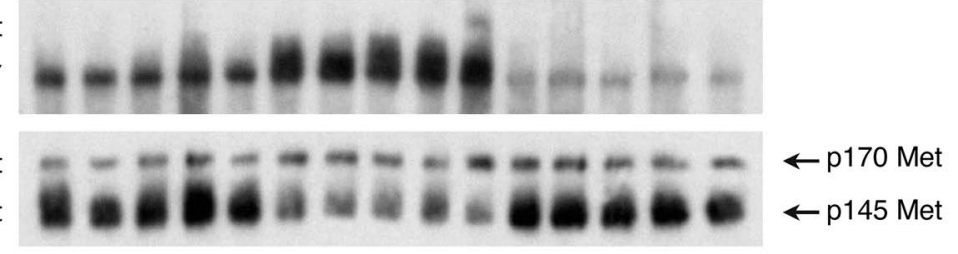

\section{Figure 8}

Expression of uncleavable SF in tumors inhibits endogenous pro-SF conversion and prevents Met activation. (A) Western blot analysis of protein extracts from lentivirus vector-transduced tumors. Upper panel: lentivirus transgene expression. Middle panel: analysis of endogenous SF activation. Lower panel: normalization of protein extracts using anti-actin. T1-T5, tumor sample number; 3T3, $\mathrm{NIH}-3 \mathrm{~T} 3$ mouse fibroblast protein extracts. (B) Analysis of Met phosphorylation in transduced tumors using anti-phosphotyrosine.

factor inhibits pro-SF conversion in vivo. Interestingly, wild-type SF also partially inhibited endogenous pro-SF processing. This is logical, as exogenous pro-SF competes with endogenous pro-SF for the active site of pro-SF convertases.

Met phosphorylation in tumors was assessed by immunoprecipitation with anti-human Met and Western blot with anti-phosphotyrosine. Tumors transduced with uncleavable SF lentivirus displayed lower Met tyrosine phosphorylation than did tumors injected with empty vector; conversely, ectopic expression of wild-type SF substantially increased receptor phosphorylation (Figure 8B).

Systemic expression of uncleavable SF suppresses tumor growth and dissemination. Systemic administration of lentivirus vectors is a powerful approach to obtain sustained plasma levels of a secreted factor (48). To this end, we i.v. injected a panel of CD-1 $\mathrm{nu}^{-/-}$mice with highly concentrated lentivirus vector preparations (empty vector, wild-type SF, or uncleavable SF) and then measured factor concentration in plasma by ELISA using specific anti-human SF. Wild-type SF consistently reached higher systemic concentrations than did uncleavable SF, although the amount of injected vector was identical (wild-type SF, $66 \pm 20$ pM; uncleavable SF, $26 \pm 9$ pM; endogenous mouse SF is 5-6 pM). However, both wild-type SF and uncleavable SF plasma levels were unexpectedly stable over time (Figure 9A).

Approximately 5 weeks after lentiviral vector injection, transduced mice were injected subcutaneously with MDAMB-435- $\beta 4$ cells, and tumor growth was monitored over time as described above. Mice transduced with uncleavable SF lentivirus were more resistant to xenograft implantation, as they developed tumors later than did control animals (mean latency: empty vector, $10 \pm 2.4$ days; uncleavable SF, $19 \pm 2.9$ days; $P=0.015$ ). Conversely, wild-type SF accelerated tumor formation (mean latency: $7.2 \pm 1.0$ days; $P=0.047)$. Tumors in mice transduced with uncleavable SF lentivirus grew significantly more slowly than control tumors, whereas the opposite was observed for tumors in mice transduced with wild-type SF lentivirus (Figure 9B). At the end of the observation period (day 48 after tumor cell injection), the mean tumor volume of mice transduced with uncleavable SF lentivirus was approximately 14 times smaller compared with mice transduced with empty vector $(P=0.002)$. Mice transduced with wild-type SF lentivirus, in contrast, bore tumors approximately 2.2-fold larger than those of control mice $(P=0.016)$.

Upon autopsy, tumors were extracted for analysis, while lungs were processed for metastasis detection as above. Analysis of tumor weight closely reflected the results obtained by volume measurement (Figure 9C). The number of macrometastases in mice injected with wild-type SF lentivirus was more than double that of control animals $(22.8 \pm 6.3$ versus $10.8 \pm 5.5 ; P=0.013)$. In contrast (Figure 9C), only one animal bore visible pulmonary lesions in the group transduced with uncleavable SF lentivirus $(0.8 \pm 1.7$; $P=0.005)$, and histological analysis revealed no micrometastases in the remaining animals (not shown).

Histological examination of tumor sections revealed significant differences among the various groups (Figure 10A). Tumors arising in animals transduced with wild-type SF vector displayed a highly invasive phenotype, demonstrated by their tendency to infiltrate adjacent tissues (e.g., muscle, adipocytes, connective tissue, mammary glands, etc.) and showed few areas of necrosis. In contrast, tumors in mice expressing uncleavable SF were well encapsulated, displayed compressive expansion rather than invasive growth toward neighboring tissues, and contained large necrotic areas relative to their reduced size. Tumors found in control animals displayed an intermediate phenotype. Tumor angiogenesis was analyzed using the CD31 endothelial marker as described above. Consistent with the previous results, 
A

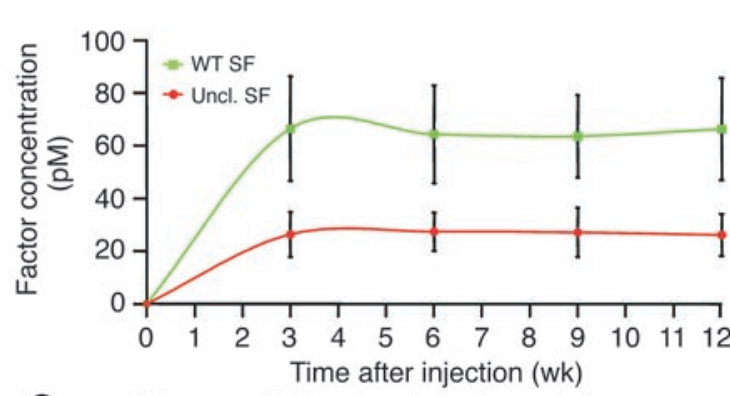

C

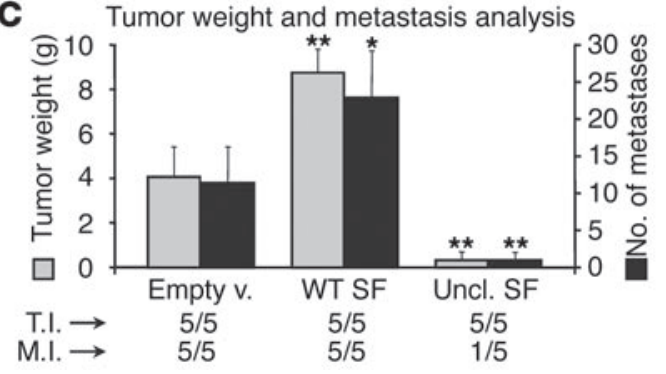

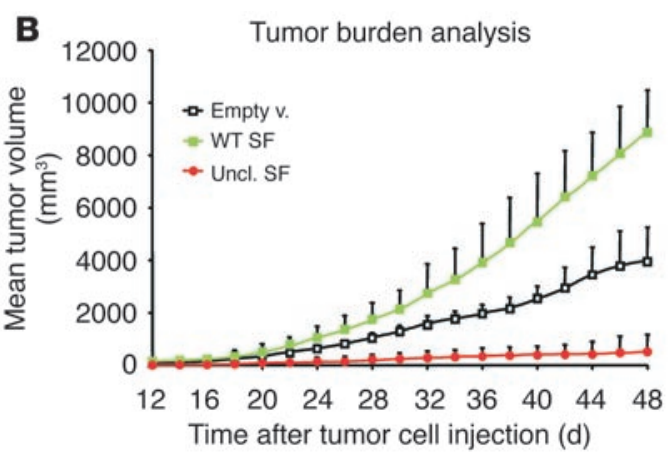

\section{Figure 9}

Lentivirus vector-mediated systemic delivery of uncleavable SF inhibits tumor growth and prevents metastatic spread. (A) Plasma levels (pmol/I) of wild-type or uncleavable SF in lentivirus vector-transduced mice, as assessed by ELISA. (B) Tumor burden analysis. Mice transduced systemically with the indicated lentivirus vectors were inoculated subcutaneously with MDA-MD-435- $\beta 4$ human mammary carcinoma cells on day 0 and were monitored every second day for tumor formation. (C) Tumor weight and metastasis analysis. Upon autopsy (on day 48), tumors were weighed (left $y$ axis, gray bars) and lungs were analyzed for the presence of metastases (right $y$ axis, black bars). Statistical significance was calculated as for Figures 5-7.

systemic expression of uncleavable SF reduced tumor vessel density by 3.5 -fold $(P=0.025)$, and wild-type SF increased it by approximately 2 -fold $(P=0.014)$. Figure $10 \mathrm{~B}$ shows representative microscopic fields of tumor vessels. Inguinal lymph nodes proximal to the tumor site almost invariably contained cancer cells. However, the extension of the metastatic area within lymph node sections varied significantly depending on the experimental group (empty vector, $20-30 \%$; wild-type SF, $60-80 \%$; uncleavable SF, 5-10\%; Figure 10C).

Effects of systemic SF/Met inbibition on "bousekeeping" physiological functions. Between 3 and 5 weeks after lentivirus vector injection, the same mice described above were subjected to a series of tests aimed at determining whether sustained systemic expression of uncleavable SF had any side effect on normal tissues or functions. We measured various parameters that could potentially be affected by SF/Met inhibition, including wound healing, hemopoiesis, blood clotting, liver markers, and kidney markers (Table 1). Upon autopsy, bone marrows were cytospun for analysis; livers and kidneys were processed for histology. Mice transduced with empty vector were used as control reference for all parameters.

Complete healing of a $5 \mathrm{~mm}$-wide, circular, transepithelial wound occurred in control mice in $9.8 \pm 1.1$ days. No statistically significant change could be detected in either of the two other groups (uncleavable SF: $11.4 \pm 1.1, P=0.053$; wild-type SF: $9.8 \pm 1.1$, $P=1.000)$. Consistent with a role for SF in hemopoiesis (21), the bone marrow cell apoptotic index was significantly increased in the group transduced with uncleavable SF lentivirus (1.4-fold; $P<0.001)$ and was substantially decreased in the group transduced with wild-type SF lentivirus $(1.3$-fold; $P<0.001)$. However, peripheral blood cell populations were normal in all mice, suggesting that the different apoptotic rate in the bone marrow was perhaps compensated by modulating proliferation. Blood clotting parameters (D-dimer test, prothrombin time, activated partial thromboplastin time, fibrinogen, and anti-thromboplastin III) and liver and kidney markers $(\gamma$-glutamyltransferase, alanine aminotransferase, blood urea nitrogen, and creatinine) were normal in all animals. Histological analysis of liver and kidney sections did not reveal any obvious signs of pathology. Organ size in all lentivirus vector-transduced mice was undistinguishable from that of untransduced mice. All animals displayed a normal feeding behavior and did not show any alteration in weight homeostasis.

\section{Discussion}

The data presented in this study demonstrate that a single-amino acid substitution in the proteolytic site of SF not only completely abrogates its ability to elicit multiple biological activities but also generates a new protein with diametrically opposed properties. In fact, while wild-type SF is a pro-invasive factor by definition, uncleavable SF potently inhibits invasive growth both in vitro and in various animal tumor models.

The first connotation of this finding is that pro-SF is therefore not just an "inactive" precursor of SF but is a precise modulator of SF activity. As previously stated, pro-SF is ubiquitous and is activated locally by target tissues. However, our data show that pro-SF competes with active SF in Met binding, suggesting that Met activation in vivo may not simply depend on the amount of active SF but rather on the ratio between active SF and pro-SF. This fine mechanism of regulation may have evolved to allow a finer tuning of invasive processes that are beneficial during embryo development, wound healing, and tissue regeneration but potentially harmful if left unchecked. 
A
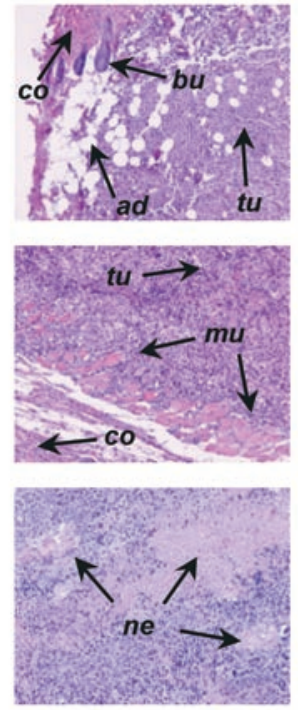

Empty v.

B

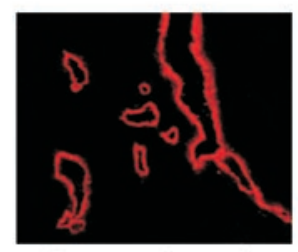

Empty v. $(81 \pm 9)$

C

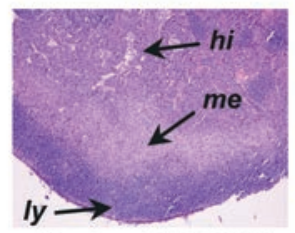

Empty v. (20-30\%)
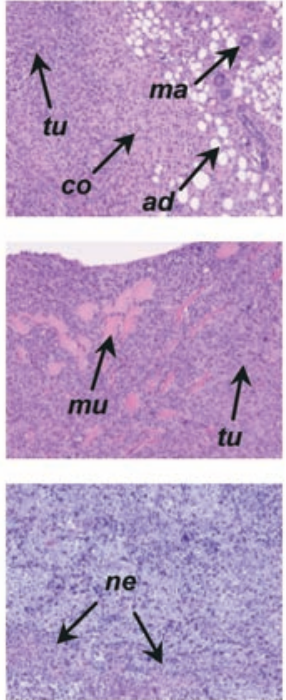

WT SF

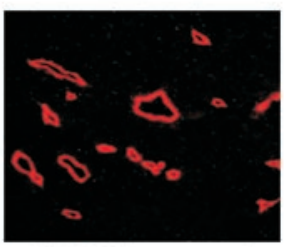

WT SF $(160 \pm 13)$

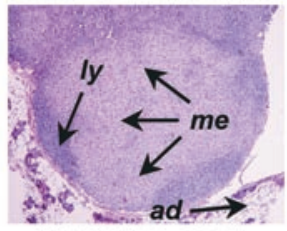

WT SF (60-80\%)
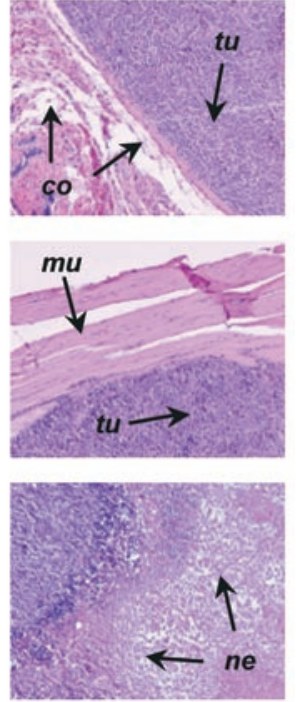

Uncl. SF

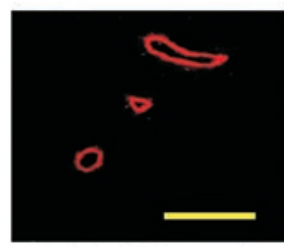

Uncl. SF $(23 \pm 4)$

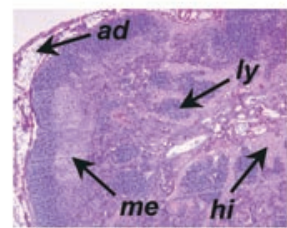

Uncl. SF (5-10\%)

Figure 10

Systemic delivery of uncleavable SF reverses the invasive phenotype of experimental tumors. (A) Histological characterization of tumors from the experiment described in Figure 9. Tumor sections stained with hematoxylin and eosin are shown at a magnification of $\times 100$. Arrows: tu, tumor cells; co, connective tissue; ad, adipocytes; bu, hair bulb; ma, mammary gland; mu, muscle; ne, necrotic tissue. (B) Immunofluorescence analysis of tumor vessels using the CD31 endothelial marker. A representative microscopic field for each experimental group is shown. Values (mean $\pm \mathrm{SD}$ ) indicate vessel density (vessels $/ \mathrm{mm}^{2}$ ) Yellow scale bar: $100 \mu \mathrm{m}$. (C) Representative hematoxylin and eosin sections of inguinal lymph nodes proximal to tumor cell injection site (magnification, $\times 50)$. Arrows: ly, lymphatic cells; hi, histiocytes; me, metastatic cells. Values refer to the percentage of lymph node section occupied by metastatic cells.

But how can unprocessed SF bind to Met with high affinity without activating it and how does active SF promote signaling? A possible explanation for this enigma is suggested by previous structure/function studies on SF/Met. There are two Met-binding sites on SF, endowed with different receptor affinity. The major binding site is on the $\alpha$-chain, while the secondary site lies within the $\beta$-chain (49). Furthermore, the $\beta$-chain may be involved in ligand-ligand interactions (50). The high-affinity site on the $\alpha$-chain is exposed in both unprocessed and active SF and dictates ligand binding; the low-affinity site becomes accessible only upon

pro-factor conversion, and while it does not substantially increase the overall affinity of the ligand, it is essential for receptor dimerization and thus activation (46, 49-52). While several molecular aspects of this mechanism are still unclear, the ability of unprocessed SF to displace active SF readily offers an attractive therapeutic opportunity.

With regard to ligand displacement, it should also be considered that active SF represents only a fraction of the total SF pool. It has been previously suggested that uPA processes pro-SF when it is already bound to Met (41). Based on our binding and cross-linking data, we can hypothesize that pro-SF is stored extracellularly not only via adsorption to heparan sulfates but also via capture by the Met receptor. The pro-SF/Met complex is quiescent, however, and becomes active only upon protease-mediated pro-SF conversion. In this scenario, uncleavable SF would therefore compete for binding mainly with pro-SF, which has equal receptor affinity. This would explain why uncleavable SF has very high inhibitory activity in vivo, despite having lower affinity for Met than active SF has.

Our results indicate that SF displacement is not the only mechanism by which uncleavable SF interferes with $\mathrm{SF} /$ Met signaling. In fact, uncleavable SF also competitively inhibited proteolytic conversion of endogenous pro-SF both in vitro and in experimental tumors. The active site of a pro-SF convertase probably cannot distinguish between wild-type pro-SF and uncleavable SF, and therefore the latter competes equally with endogenous pro-SF for entry into the catalytic pocket. Some proteases involved in pro-SF conversion have a broad range of activity (e.g., uPA), and therefore uncleavable SF could in principle compete with other substrates as well. However, our experiments using Met-negative xenografts demonstrated that uncleavable SF has no effect on tumors that do not express the SF receptor. Therefore, either protease inhibition on its own is not sufficient to suppress tumor growth or uncleavable SF selectively inhibits pro-SF conversion. In any case, these data suggest that tumor inhibition by uncleavable SF depends on functional SF/Met signaling on tumor cells.

The ability of uncleavable SF to combine ligand-displacement activity with prevention of wild-type pro-SF conversion sets it apart from previously developed inhibitors of SF/Met, including NK4 HGF (competitive SF antagonist; ref. 43), monoclonal antibodies against SF (ligand-neutralizing agents; ref. 42), and decoy Met (ligand neutralizer and dominant negative inhibitor; ref. 44). These molecules interfere with SF/Met signaling at the level of ligand-receptor binding and/or receptor-receptor interaction. Uncleavable SF, by inhibiting pro-SF convertase activity, achieves an "autocatalytic" effect: it reduces the amount of active SF and increases the levels of unprocessed SF. The latter, in turn, may contribute to the displacement of active SF from Met.

Our preclinical experiments suggest that uncleavable SF has a significant antitumor activity in experimental animals and, perhaps most relevantly, that it completely suppresses the formation of metastases in both local and systemic delivery protocols. What may be the mechanisms underlying this inhibition and what are the biological processes involved? 


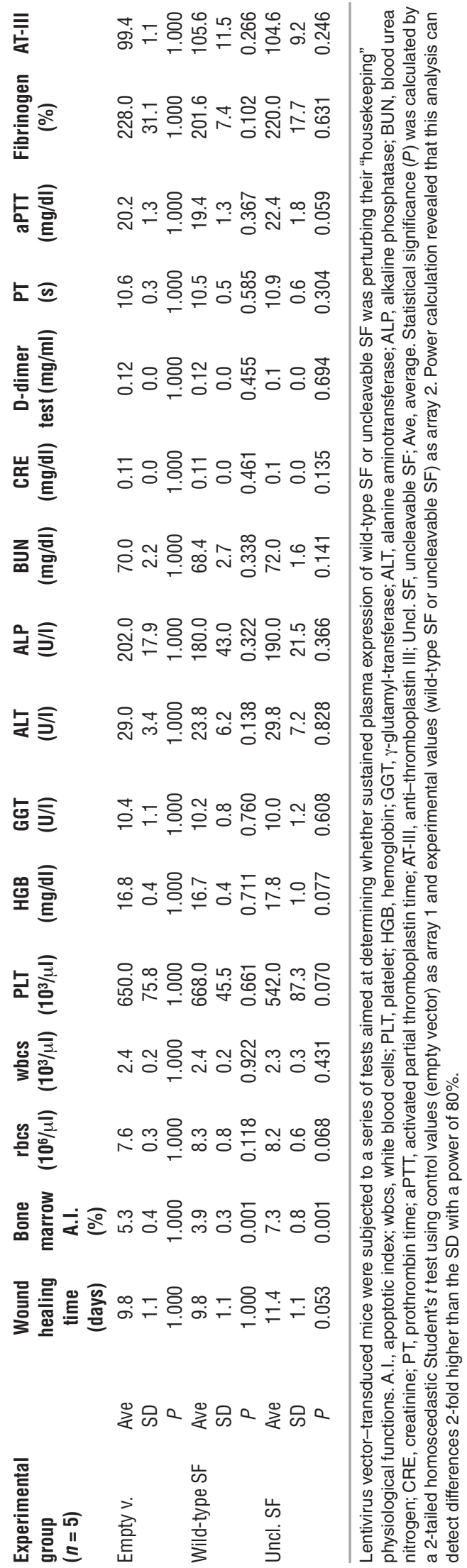

The foundation of uncleavable SF-mediated tumor suppression lies in its ability to interfere with the signals exchanged between the host and the tumor. In fact, rather than being passive bystanders, host tissues often provide critical support to the trophism and dissemination of cancer cells via a complex network of cytokines, growth factors, and hormones. SF is a key component of this network: it is produced by host mesenchymal cells and it regulates proliferation, scattering, migration, survival, and morphogenesis on a broad range of Met-expressing tissues, including both tumor cells and host endothelial cells. In the case of a carcinoma (the model used in this study), SF sustains cancer cell proliferation, inhibits apoptosis, and induces extracellular matrix remodeling. Tumor cells are stimulated by SF to loosen their interactions with neighboring epithelial cells and to migrate through the basement membrane toward the contiguous connective tissue. Once surrounded by stromal cells, a carcinoma finds itself in a foreign environment, and SF protects it against "anoikis" (54). Consistent with this, uncleavable SF inhibited cell proliferation, increased apoptotic cell death, and inhibited local tumor invasion. SF also attracts new blood and lymph vessels, which not only supply the tumor with oxygen and nutrients but also serve as "escape routes" for metastatic cells. In fact, local or systemic delivery of uncleavable SF impaired tumor angiogenesis and reduced lymph node colonization, while wild-type SF elicited the opposed effect. Once in circulation, a metastatic cell still has to arrest at a secondary site, to extravasate, and to implant in a new tissue, where it must survive, proliferate, and generate its own network of capillaries. In these processes, too, SF provides a critical support: it promotes cell survival and extravasation and sustains proliferation and vascularization of micrometastases. Indeed, uncleavable SF almost completely abolished the ability of i.v. injected tumor cells to form detectable pulmonary colonies, while SF caused the formation of large lung metastatic lesions.

In conclusion, tumor progression and metastasis are multistep processes that benefit from SF signaling in several of their many stages. From a pharmacological viewpoint, we are used to thinking of drugs as specific agents that target a limiting step along a pathological pathway. Uncleavable SF, in contrast, targets a pleiotropic cytokine that controls this pathological pathway at many different intermediate points, although perhaps none of them is strictly SF dependent. The two strategies are definitely symmetrical from a philosophical viewpoint, but our results demonstrate that the latter, "global" approach can be very effective therapeutically, at least in experimental systems.

In the systemic delivery approach, chronic exposure to uncleavable SF did not significantly perturb any of the analyzed physiological function, while it notably reduced tumor growth and nearly completely suppressed metastatic spread. There are several aspects of this observation that merit comment. Above all, our analysis of clinical parameters is preliminary and was conducted using a limited number of animals. We cannot rule out the possibility that a more thorough analysis performed on a larger experimental population would unmask more subtle changes escaping our examination. Furthermore, in a systemic gene transfer approach, cells expressing amounts of inhibitor that are too high (and thus toxic) may be counter-selected. In fact, uncleavable SF consistently reached lower plasma levels than wild-type SF, even though the amount of lentivirus vector injected was identical. However, these levels of inhibitor, while not affecting "housekeeping" functions, were sufficient to prevent tumor growth and 
dissemination, thus demonstrating that tumors rely on SF/Met signaling more than normal tissues do. This notion is in line with a recent report using a different SF/Met inhibitor (44).

What is the biological rationale behind this differential sensitivity? The key point is that invasive growth is not a "housekeeping" physiological function but is confined temporally and spatially to specific biological processes, including embryo development and tissue regeneration. In normal circumstances, invasive growth does not take place, and therefore SF/Met signaling may be dispensable. An actively growing tumor, in contrast, recapitulates embryo development in many of its features (e.g., absence of stromal-epithelial barriers, dynamic remodeling of tissue architecture, lively angiogenesis, migration of cells outside their "home" environment, widespread tissue hypoxia, recruitment of circulating stem cells, and active proliferation). These biological processes are SF/Met dependent and therefore offer an interesting opportunity for therapeutic intervention using SF/Met antagonists.

\section{Methods}

Factor engineering. The exact amino acid sequence of uncleavable SF corresponds to the sequence of GenBank accession number M73239 with a R489Q substitution and a poly-His tag (DDDKHHHHHH) at the C terminus. The same poly-His tag was added to wild-type, human SF. Myctagged SF contains a Myc epitope (EQKLISEEDLN) in addition to the above poly-His tag. All cDNAs were subcloned into the lentivirus transfer vector pRRLsin.PPT.CMV.eGFP.Wpre (45) in place of the g fp cDNA.

Lentivirus vector preparation and in vitro cell transduction. Vector stocks were produced as described previously (55). The concentration of viral p24 antigen was assessed using the HIV-1 p24 core profile ELISA kit (NEN Life Science Products) according to the manufacturer's instructions. Cells were transduced in six-well plates ( $10^{5}$ cells/well in $2 \mathrm{ml}$ of medium) using $40 \mathrm{ng} /$ $\mathrm{ml}$ of p24 in the presence of $8 \mu \mathrm{g} / \mathrm{ml}$ polybrene (Sigma) as described (55).

Ex vivo tumorigenesis assays. Lentivirus vector-transduced tumor cells were injected subcutaneously into the right posterior flanks of 6-week old immunodeficient $n u^{-/}$female mice on a Swiss CD-1 background (Charles River Laboratories). The number of cells injected per mouse varied by cell line (MDA-MB-435 cells, $2 \times 10^{6}$; MDA-MB- $435-\beta 4$ cells, $1 \times 10^{6}$; A549 cells, $4 \times 10^{6}$; HCT- 116 cells, $2 \times 10^{6}$; TOV- $112 \mathrm{D}$ cells, $\left.6 \times 10^{6}\right)$. Tumor volume was calculated as described (44). At the end of the observation period, tumors were weighed, and lung nodules were contrasted with black India ink to facilitate assignment of scores. Superficial pulmonary metastases were counted under a stereoscopic microscope. Histological analysis was performed on paraffin-embedded sections stained with hematoxylin and eosin. All animal procedures were approved by the Ethical Commission of the University of Torino, Italy, and by the Italian Ministry of Health.

Direct injection of lentivirus vectors into preformed tumors. MDA-MB-435- $\beta 4$ tumor cells were injected subcutaneously into immunodeficient mice as described above $\left(2 \times 10^{6}\right.$ cells/mouse). After 2 weeks, mice bearing tumors of approximately $100 \mathrm{~mm}^{3}$ were selected and were randomly divided into 3 groups of 6 mice each. At this time (day 0 ) and on days 3, 9, and 15, hightiter lentivirus vector preparations ( $1 \mu \mathrm{g}$ p $24 /$ mouse) were administrated intratumorally using a Hamilton syringe (Hamilton). Tumor volume was measured every 3 days. At day 18, mice were killed by $\mathrm{CO}_{2}$ inhalation; tumors were excised and weighed. Half of each tumor was embedded in Tissue-Tek OCT compound (Sakura Finetek) and was immediately frozen in liquid nitrogen. Frozen tumor sections were used for tissue analysis. The other half of each tumor was used for preparation of protein extracts. Lungs were contrasted with India ink as described above, analyzed by microscopy, and then processed for histological analysis.

Systemic delivery of lentivirus vectors. Six-week-old nude female mice (6 mice) group) were injected through the tail vein with concentrated lentivirus vector (20 $\mu \mathrm{g}$ p 24/mouse). After 3 weeks, plasma concentrations of recombinant proteins were estimated by ELISA (see Supplemental Methods; supplemental material available at http://www.jci.org/cgi/content/full/114/10/1418/DC1). Mice expressing the lowest levels of factors were excluded (1 mouse/group). MDA-MB-435- $\beta 4$ cells were injected subcutaneously as described above and tumor burden was measured every 2 days. During this period, blood samples were obtained periodically, and plasma factor concentrations as well as a series of clinical parameters were assessed. After an observation period of 48 days, mice were killed and subjected to autopsy. Lungs were stained with India ink to contrast lung nodules and then processed for histological analysis. Tumors were excised and weighed. Half of each tumor was embedded in Tissue-Tek OCT compound and was immediately frozen in liquid nitrogen; the other half was embedded in paraffin. Right inguinal lymph nodes were collected and embedded in paraffin. Bone marrow was harvested from femora and was suspended in PBS plus 2\% FBS for cytological analysis. Livers and kidneys were embedded in paraffin and were processed for histological analysis.

Additional methods. For all experimental procedures not listed here, please refer to the online Supplemental Methods section.

\section{Acknowledgments}

We are grateful to Antonino Sottile for analysis of clinical parameters, Giovanni De Rosa for histological consultation, Giuliana Strola for bone marrow analysis, Francesco Blasi for antibodies against uPA, Paolo Ferrero and Graziano Carchia for tissue processing, and Ada Arnesano for skilled technical assistance. This work has been supported in part by the Italian Association for Cancer Research, the Compagnia San Paolo di Torino Foundation, and the Italian Ministry of University and Research (grant number 2001068458).

Received for publication May 24, 2004, and accepted in revised form September 14, 2004.

Address correspondence to: Paolo Michieli, Institute for Cancer Research and Treatment (IRCC), Division of Molecular Oncology, University of Torino Medical School, Strada Provinciale 142, km 3.95, I-10060 Candiolo (Torino), Italy. Phone: 39-011-9933-219; Fax: 39-011-9933-225; E-mail: paolo.michieli@ircc.it.

Luigi Naldini's present address is: San Raffaele Telethon Institute for Gene Therapy (HSR-TIGET), Milano, Italy.
1. Stoker, N., Gherardi, E., Perryman, M., and Grey, J. 1987. Scatter factor is a fibroblast-derived modulator of epithelial cell motility. Nature. 327:239-242.

2. Gherardi, E., Gray, J., Stoker, M., Perryman, M., and Furlong, R. 1989. Purification of scatter factor, a fibroblast-derived basic protein that modulates epithelial interactions and movement. Proc. Natl. Acad. Sci. U. S. A. 86:5844-5848.

3. Nakamura, T., Teramoto, H., and Ichihara, A. Purification and characterization of a growth factor from rat platelets for mature parenchymal hepatocytes in primary cultures. 1986. Proc. Natl. Acad. Sci. U. S. A. 83:6489-6493.

4. Zarnegar, R., and Michalopoulos, G. 1989. Purification and biological characterization of human hepatopoietin A, a polypeptide growth factor for hepatocytes. Cancer Res. 49:3314-3320.

5. Weidner, K.M., et al. 1991. Evidence for the identity of human scatter factor and human hepatocyte growth factor. Proc. Natl. Acad. Sci. U. S. A.
88:7001-7005.

6. Naldini, L., et al. 1991. Scatter factor and hepatocyte growth factor are indistinguishable ligands for the MET receptor. EMBO J. 10:2867-2878.

7. Montesano, R., Matsumoto, K., Nakamura, T., and Orci L. 1991. Identification of a fibroblast-derived epithelial morphogen as hepatocyte growth factor. Cell. 67:901-908.

8. Rubin, J.S., Bottaro, D.P., and Aaronson, S.A. 1993. Hepatocyte growth factor/scatter factor and 
its receptor, the c-met proto-oncogene product. Biochim. Biophys. Acta. 1155:357-371.

9. Zarnegar, R., and Michalopoulos, G. 1995. The many faces of hepatocyte growth factor: from hepatopoiesis to hematopoiesis. J. Cell Biol. 129:1177-1180.

10. Tamagnone, L., and Comoglio, P.M. 1997. Control of invasive growth by hepatocyte growth factor (SF) and related scatter factors. Cytokine Growth Factor Rev. 8:129-142.

11. Comoglio, P.M., and Trusolino, L. 2002. Invasive growth: from development to metastasis. J. Clin. Invest. 109:857-862. doi:10.1172/JCI200215392.

12. Trusolino, L., and Comoglio, P.M. 2002. Scatterfactor and semaphorin receptors: cell signalling for invasive growth. Nat. Rev. Cancer. 4:289-300.

13. Woolf, A.S., et al. 1995. Roles of hepatocyte growth factor/scatter factor and the met receptor in the early development of the metanephros. J. Cell Biol. 128:171-184

14. Takayama, H., La Rochelle, W.J., Anver, M., Bockman, D.E., and Merlino, G. 1996. Scatter factor/ hepatocyte growth factor as a regulator of skeletal muscle and neural crest development. Proc. Natl. Acad. Sci. U. S. A. 93:5866-5871.

15. Andermarcher, E., Surani, M., and Gherardi, E. 1996. Co-expression of the SF/HGF and c-met genes during early mouse embryogenesis precedes reciprocal expression in adjacent tissues during organogenesis. Dev. Genet. 18:254-266

16. Bladt, F., Riethmacher, D., Isenmann, S., Aguzzi, A., and Birchmeier, C. 1995. Essential role for the c-met receptor in the migration of myogenic precursor cells into the limb bud. Nature. 376:768-771.

17. Schmidt, C., et al. 1995. Scatter factor/hepatocyte growth factor is essential for liver development Nature. 373:699-702.

18. Uehara, Y., et al. 1995. Placental defect and embryonal lethality in mice lacking hepathocyte growth factor/scatter factor. Nature. 373:702-705

19. Huh, C.G., et al. 2004. Hepatocyte growth factor/ c-met signaling pathway is required for efficient liver regeneration and repair. Proc. Natl. Acad. Sci. U. S. A. 101:4477-4482.

20. Yoshida, S., et al. 2003. Neutralization of hepatocyte growth factor leads to retarded cutaneous wound healing associated with decreased neovascularization and granulation tissue formation. J. Invest. Dermatol. 120:335-343.

21. Galimi, F., et al. 1994. Hepatocyte growth factor induces proliferation and differentiation of multipotent and erythroid hemopoietic progenitors. J. Cell Biol. 127:1743-1754.

22. Birchmeier, C., Birchmeier, W., Gherardi, E., and Vande Woude, G.F. 2003. Met, metastasis, motility, and more. Nat. Rev. Mol. Cell Biol. 4:915-925.

23. Naldini, L., et al. 1992. Extracellular proteolytic cleavage by urokinase is required for activation of hepatocyte growth factor/scatter factor. $E M B O J$. 11:4825-4833

24. Naka, D., et al. 1992. Activation of hepatocyte growth factor by proteolytic conversion of a single chain form to a heterodimer. J. Biol. Chem.
267:20114-20119.

25. Kobayashi, T., et al. 1994. Hepatocyte growth factor specifically binds to sulfoglycolipids. J. Biol. Chem 269:9817-9821.

26. Lyon, M., Deakin, J.A., Mizuno, K., Nakamura, T., and Gallagher J.T. 1994. Interaction of hepatocyte growth factor with heparan-sulfate. Elucidation of the major heparan sulfate structural determinants. J. Biol. Chem. 269:11216-11223.

27. Zhu, Y., Hojo, Y., Ikeda, U., and Shimada, K. 2000. Production of hepatocyte growth factor during acute myocardial infarction. Heart. 83:450-455.

28. Shiota, G., Umeki, K., Okano, J., and Kawasaki, H. 1995. Hepatocyte growth factor and acute phase proteins in patients with chronic liver diseases. J. Med. 26:295-308

29. Nakamura, S., Gohda, E., Matsuo, Y., Yamamoto, I., and Minowada, J. 1994. Significant amount of hepatocyte growth factor detected in blood and bone marrow plasma of leukaemia patients. $\mathrm{Br}$. J. Haematol. 87:640-642.

30. Miyazawa, K., Shimomura, T., Naka, D., and Kitamura, N. 1994. Proteolytic activation of hepatocyte growth factor in response to tissue injury. J. Biol. Chem. 269:8966-8970.

31. Mizuno, K., Takehara, T., and Nakamura, T. 1992. Proteolytic activation of a single-chain precursor of hepatocyte growth factor by extracellular serine-protease. Biochem. Biophys. Res. Commun. 189:1631-1638.

32. Peek, M., Moran, P., Mendoza, N., Wickramasinghe, D., and Kirchhofer, D. 2002. Unusual proteolytic activation of pro-hepatocyte growth factor by plasma kallikrein and coagulation factor XIa. J. Biol. Chem. 277:47804-47809.

33. Shimomura, T., et al. 1993. Activation of the zymogen of hepatocyte growth factor activator by thrombin. J. Biol. Chem. 268:22927-22932.

34. Schmassmann, A., et al. 1997. Roles of hepatocyte growth factor and its receptor Met during gastric ulcer healing in rats. Gastroenterology. 113:1858-1872.

35. Jiang, W.G., and Harding, K.G. 1998. Enhancement of wound tissue expansion and angiogenesis by matrix-embedded fibroblast (dermagraft), a role of hepatocyte growth factor/scatter factor. Int. J. Mol. Med. 2:203-210.

36. Sugimura, K., et al. 2001. Production and activation of hepatocyte growth factor in acute renal failure. Ren. Fail. 23:597-603.

37. Aguirre Ghiso, J.A., Alonso, D.F., Farias, E.F., Gomez, D.E., and de Kier Joffe, E.B. 1999. Deregulation of the signaling pathways controlling urokinase production. Its relationship with the invasive phenotype. Eur. J. Biochem. 263:295-304.

38. Parr, C., Watkins, G., Mansel, R.E., and Jiang, W.G. 2004. The hepatocyte growth factor regulatory factors in human breast cancer. Clin. Cancer Res. 10:202-211.

39. Pennacchietti, S., et al. 2003. Hypoxia promotes invasive growth by transcriptional activation of the met protooncogene. Cancer Cell. 3:347-361.

40. Graham, C.H., Fitzpatrick, T.E., and McCrae, K.R. 1998. Hypoxia stimulates urokinase recep- tor expression through a heme protein-dependent pathway. Blood. 91:3300-3307.

41. Naldini, L., et al. 1995. Biological activation of pro-HGF (hepatocyte growth factor) by urokinase is controlled by a stoichiometric reaction. J. Biol. Chem. 270:603-611.

42. Cao, B., et al. 2001. Neutralizing monoclonal antibodies to hepatocyte growth factor/scatter factor (HGF/SF) display antitumor activity in animal models. Proc. Natl. Acad. Sci. U. S. A. 98:7443-7448.

43. Matsumoto, K., and Nakamura, T. 2003. NK4 (HGF-antagonist/angiogenesis inhibitor) in cancer biology and therapeutics. Cancer Sci. 94:321-327.

44. Michieli, P., et al. 2004. Targeting the tumor and its microenvironment by a dual-function decoy Met receptor. Cancer Cell. 6:61-73.

45. Follenzi, A., Ailles, L.E., Bakovic, S., Geuna, M., and Naldini, L. 2000. Gene transfer by lentiviral vectors is limited by nuclear translocation and rescued by HIV-1 pol sequences. Nat. Genet. 25:217-222.

46. Lokker, N.A., et al. 1992. Structure-function analysis of hepatocyte growth factor: identification of variants that lack mitogenic activity yet retain high affinity receptor binding. EMBO J. 11:2503-2510.

47. Bussolino, F., et al. 1992. Hepatocyte growth factor is a potent angiogenic factor which stimulates endothelial cell motility and growth. J. Cell Biol. 119:629-641

48. Follenzi, A., Sabatino, G., Lombardo, A., Boccaccio, C., and Naldini, L. 2002. Efficient gene delivery and targeted expression to hepatocytes in vivo by improved lentiviral vectors. Hum. Gene Ther. 13:243-260.

49. Matsumoto, K., Kataoka, H., Date, K., and Nakamura, T. 1998. Cooperative interaction between alpha- and beta-chains of hepatocyte growth factor on c-Met receptor confers ligand-induced receptor tyrosine phosphorylation and multiple biological responses. J. Biol. Chem. 273:22913-22920.

50. Stamos, J., Lazarus, R.A., Yao, X., Kirchhofer, D., and Wiesmann, C. 2004. Crystal structure of the HGF beta-chain in complex with the Sema domain of the Met receptor. EMBOJ. 23:2325-2335.

51. Kirchhofer, D., et al. 2004. Structural and functional basis of the serine protease-like HGF betachain in Met binding and signaling. J. Biol. Chem. 279:39915-39924.

52. Hartmann, G., et al. 1992. A functional domain in the heavy chain of scatter factor/hepatocyte growth factor binds the c-Met receptor and induces cell dissociation but not mitogenesis. Proc. Natl. Acad. Sci.U.S. A. 89:11574-11578.

53. Choong, P.F., and Nadesapillai, A.P. 2003. Urokinase plasminogen activator system: a multifunctional role in tumor progression and metastasis. Clin. Orthop. 415:S46-S58.

54. Frisch, S.M., and Francis, H. 1994. Disruption of epithelial cell-matrix interactions induces apoptosis. J. Cell Biol. 124:619-626.

55. Vigna, E., and Naldini, L. 2000. Lentiviral vectors: excellent tools for experimental gene transfer and promising candidates for gene therapy. J. Gene Med. 2:308-316. 